\title{
U.S. Hydropower Resource Assessment for Tennessee
}

Prepared by: Alison M. Conner James E. Francfort

Project Manager:

Ben N. Rinehart

Published July 1997

Idaho National Engineering and Environmental Laboratory Renewable Energy Products Department Lockheed Idaho Technologies Company Idaho Falls, Idaho 83415

Prepared for the

U.S. Department of Energy

Assistant Secretary for Energy Efficiency and Renewable Energy

Under DOE Idaho Operations Office

Contract DE-AC07-94ID13223 


\section{DISCLAIMER}

Portions of this document may be illegible electronic image products. Images are produced from the best available original document. 


\begin{abstract}
The U.S. Department of Energy is developing an estimate of the undeveloped hydropower potential in the United States. The Hydropower Evaluation Software (HES) is a computer model that was developed by the Idaho National Engineering Laboratory for this purpose. HES measures the undeveloped hydropower resources available in the United States, using uniform criteria for measurement. The software was developed and tested using hydropower information and data provided by the Southwestern Power Administration. It is a menu-driven program that allows the personal computer user to assign environmental attributes to potential hydropower sites, calculate development suitability factors for each site based on the environmental attributes present, and generate reports based on these suitability factors. This report describes the resource assessment results for the State of Tennessee.
\end{abstract}

\title{
DISCLAIMER
}

This report was prepared as an account of work sponsored by an agency of the United States Government. Neither the United States Government nor any agency thereof, nor any of their employees, makes any warranty, express or implied, or assumes any legal liability or responsibility for the accuracy, completeness, or usefulness of any information, apparatus, product, or process disclosed, or represents that its use would not infringe privately owned rights. Reference herein to any specific commercial product, process, or service by trade name, trademark, manufacturer, or otherwise does not necessarily constitute or imply its endorsement, recommendation, or favoring by the United States Government or any agency thereof. The views and opinions of authors expressed herein do not necessarily state or reflect those of the United States Government or any agency thereof. 


\section{ACKNOWLEDGMENTS}

The authors thank Peggy A. M. Brookshier and John V. Flynn of the U.S. Department of Energy, and Lyle Bentley of the State of Tennessee for their active participation and timely comments. 


\section{CONTENTS}

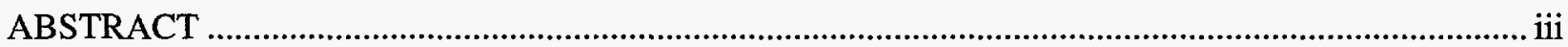

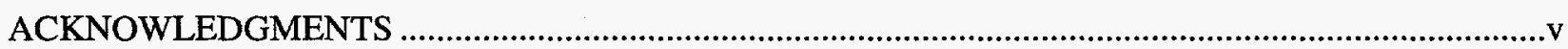

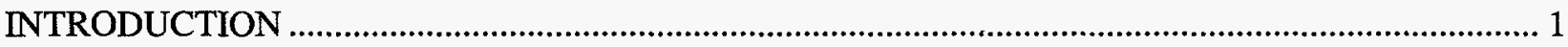

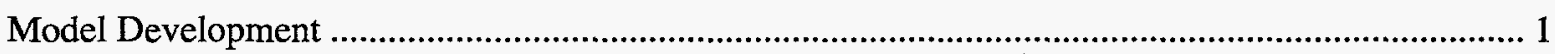

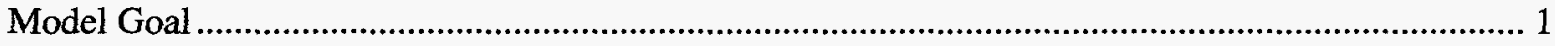

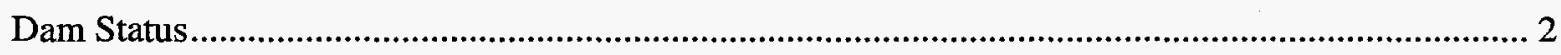

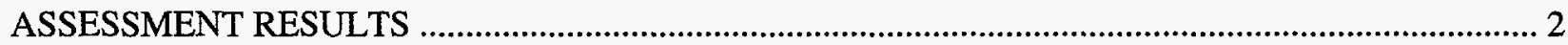

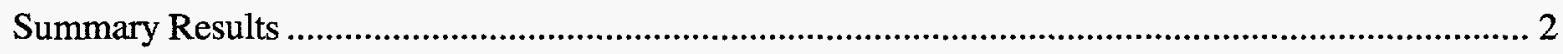

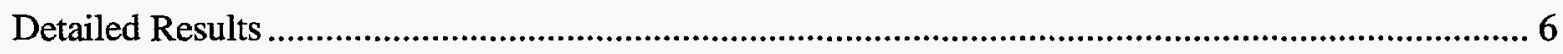

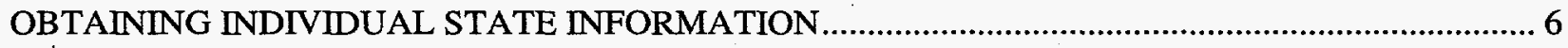

ADDITIONAL HYDROPOWER EVALUATION SOFTWARE INFORMATION................................ 7

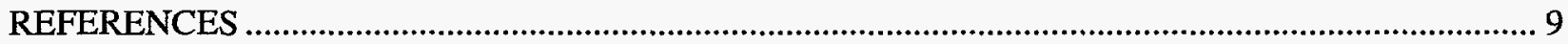

Appendix A-Summary Report

Appendix B-River Basins Report

Appendix C-Tennessee Sites List

Appendix D-Individual Resource Database List

\section{FIGURES}

1. Number of sites, by capacity groups, with HES-modeled undeveloped hydropower potential ............ 3

2. The nonmodeled and HES-modeled undeveloped hydropower potential ...................................... 3

3. The number of sites with undeveloped hydropower potential and the total megawatts of HES-modeled undeveloped hydropower potential. 
4. The Burgess Falls Dam in the Burgess Falls State Natural Area ............................................... 5

5. The Fairfield Mill Dam in Bedford County, Tennessee............................................................... 5

6. Number of sites with undeveloped hydropower potential in the Tennessee river basins .................... 6

7. Megawatts of HES-modeled undeveloped hydropower potential in the Tennessee river basins.......... 6

\section{TABLES}

1. Undeveloped hydropower potential summary for Tennessee. 2 


\section{U.S. Hydropower Resource Assessment for Tennessee}

\section{INTRODUCTION}

In June 1989, the U.S. Department of Energy initiated the development of a National Energy Strategy to identify the energy resources available to support the expanding demand for energy in the United States. Public hearings conducted as part of the strategy development process indicated that undeveloped hydropower resources were not well defined. As a result, the Department of Energy established an interagency Hydropower Resource Assessment Team to ascertain the undeveloped hydropower potential. In connection with these efforts by the Department of Energy, the Idaho National Engineering Laboratory designed the Hydropower Evaluation Software (HES), which has been used to perform a resource assessment of the undeveloped conventional hydropower potential in over 30 states. This report presents the results of the hydropower resource assessment for the State of Tennessee. Undeveloped pumped storage hydropower potential is not included.

The HES was developed as a tool to measure undeveloped hydropower potential regionally or by state. The software is not intended to provide precise development factors for individual sites, but to provide regional or state totals. Because the software was developed as a generic measurement tool encompassing national issues, regional and state totals must be considered judiciously; various local issues may skew undeveloped hydropower potential totals. The information for the resource assessment was compiled from the Federal Energy Regulatory Commission's Hydroelectric Power Resources Assessment database and several other sources. Refer to DOE/ID-10338, the User's Manual (Francfort, Matthews, Rinehart 1991) for the specifics of the software and to DOE/D-10430.1, the Status Report (Conner, Francfort, Rinehart 1996) for an overview of all resource assessment activities to date.

\section{Model Development}

Hydropower Evaluation Software, both a probability-factor computer model and a database, is a menu-driven program that is intended to be user-friendly. Computer screens and report-generation capabilities were developed to meet the needs of users nationwide. The software uses environmental attribute data to generate an overall project environmental suitability factor (PESF) between 0.1 and 0.9 , where 0.9 indicates the highest likelihood of development and 0.1 indicates the lowest likelihood of development. The suitability factors are dependant on the unique environmental attributes of each potential site. They reflect the considerations that (a) environmental concerns can make a potential site unacceptable, prohibiting its development (for a suitability factor of 0.1 ), or (b) if there are no environmental concerns, there is no negative effect on the likelihood of site development (for a suitability factor of 0.9 ). A combination of attributes can result in a lower suitability factor because multiple environmental considerations would reduce the likelihood that a site may be developed to its physical potential.

\section{Model Goal}

The goal of the HES is to assemble an accurate resource database of all sites with undeveloped hydropower potential in the United States for use as a planning tool to determine the viable national hydropower potential. Undeveloped hydropower potential is not limited to the development of new sites; it also includes the development of additional hydropowergenerating capacity at sites that currently have hydropower, but are not developed to their full potential. This undeveloped hydropower potential is a source of nonpolluting, renewable energy available to meet the growing power needs of the United States. The HES should help make this goal obtainable and ensure a set of uniform criteria for national assessment. 


\section{Dam Status}

The effects of environmental attributes vary by dam status. The dam status classifications used are as follows:

$$
\begin{aligned}
& \mathrm{W}=\text { Developed hydropower site } \\
& \text { with current power generation, } \\
& \text { but the total hydropower } \\
& \text { potential has not been fully } \\
& \text { developed. Only the } \\
& \text { undeveloped hydropower } \\
& \text { potential is discussed in this } \\
& \text { report. }
\end{aligned}
$$

$\mathrm{W} / \mathrm{O}=$ Developed site without current power generation. The site has some type of developed impoundment or diversion structure, but no developed hydropower generating capability.

$\mathrm{U}=$ Undeveloped site. The site does not have power generation capability nor a developed impoundment or diversion structure.

\section{ASSESSMENT RESULTS Summary Results}

A total of 22 sites (Table 1) have been identified and assessed for their undeveloped hydropower potential. The HES results for individual site capacities range from 9 kilowatts (kW) to 90 megawatts (MW). About one-half the sites are less than $1 \mathrm{MW}$, three are micro sites less than $100 \mathrm{~kW}$, and the other half or greater than 1 MW, one of which is an undeveloped site on the Mississippi River with an estimated potential capacity of $90 \mathrm{MW}$ (Figure 1).

The nonmodeled undeveloped hydropower potential total for Tennessee was identified as 496 MW. The HES results lowers this estimate about $72 \%$ to $138 \mathrm{MW}$. The greatest reduction in undeveloped hydropower potential, by MW, occurs at sites with no current power generation capability nor impoundment or diversion structure in place (undeveloped category). These sites have an HES-modeled undeveloped hydropower potential of $128 \mathrm{MW}$, a $348 \mathrm{MW}$ reduction in the estimated undeveloped hydropower potential (Figure 2). The number of sites does not change, only the identified undeveloped hydropower potential is reassessed (Figure 3). Figures 4 and 5 illustrate two historic dam sites in Tennessee, the Burgess Falls Dam and the Fairfield Mill Dam, neither of which produce power.

The 22 identified sites are located within 3 major river basins. The number of sites per major river basin ranges from 1 in the St. Francis River Basin to 16 in the Tennessee River Basin (Figure 6). The St. Francis River Basin, with only 1 identified site, has the most undeveloped hydropower potential, $90 \mathrm{MW}$ or $65 \%$ of the total undeveloped hydropower potential, of the Tennessee river basins (Figure 7).

Table 1. Undeveloped hydropower potential summary for Tennessee. The table contains the nonmodeled undeveloped nameplate potential and the HES-modeled undeveloped potential totals.

\begin{tabular}{lccc}
\hline & Number of projects & $\begin{array}{r}\text { Nameplate potential } \\
\text { (MW) }\end{array}$ & $\begin{array}{c}\text { HES-modeled potential } \\
\text { (MW) }\end{array}$ \\
\cline { 2 - 4 } With Power & 0 & 0 & 0 \\
W/O Power & 11 & 20.0 & 10.0 \\
Undeveloped & 11 & 475.9 & 127.6 \\
State Total & 22 & 495.9 & 137.6 \\
\hline
\end{tabular}




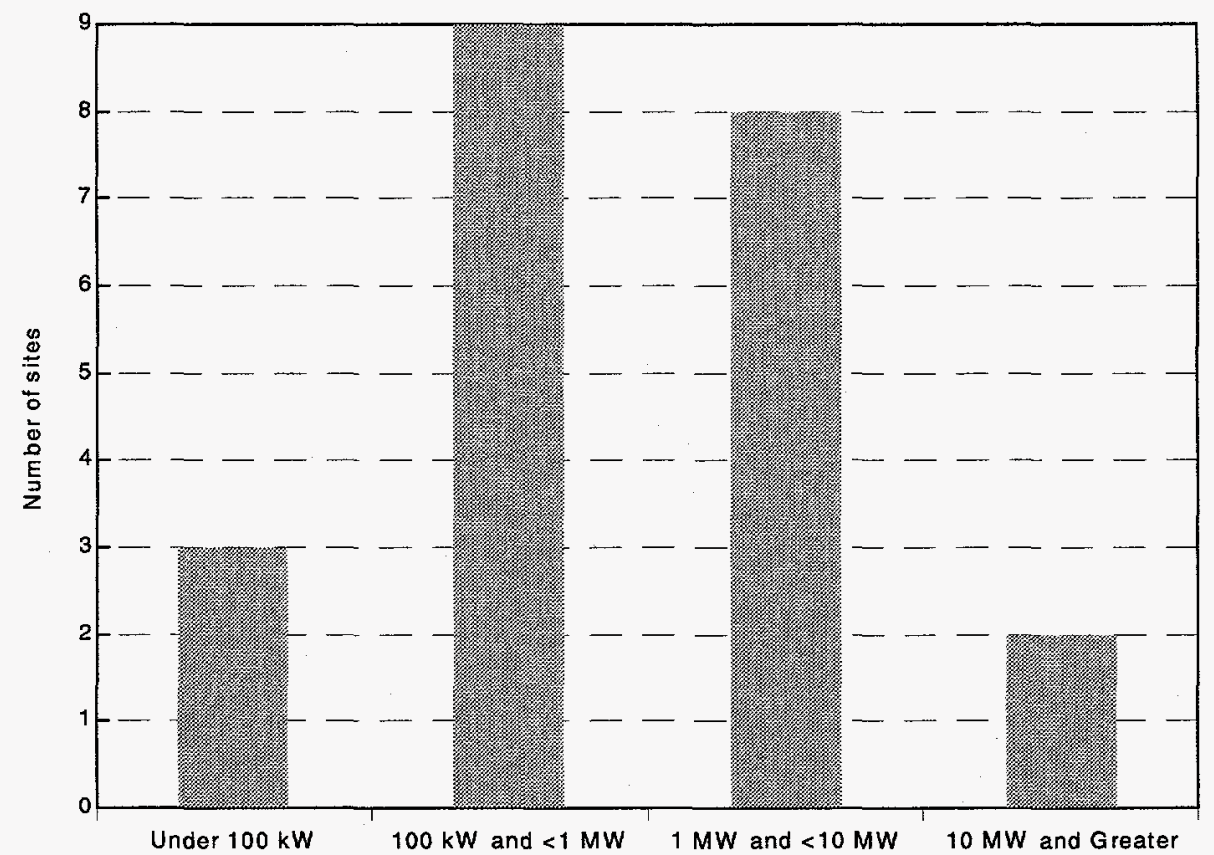

Figure 1. Number of sites, by capacity groups, with HES-modeled undeveloped hydropower potential.

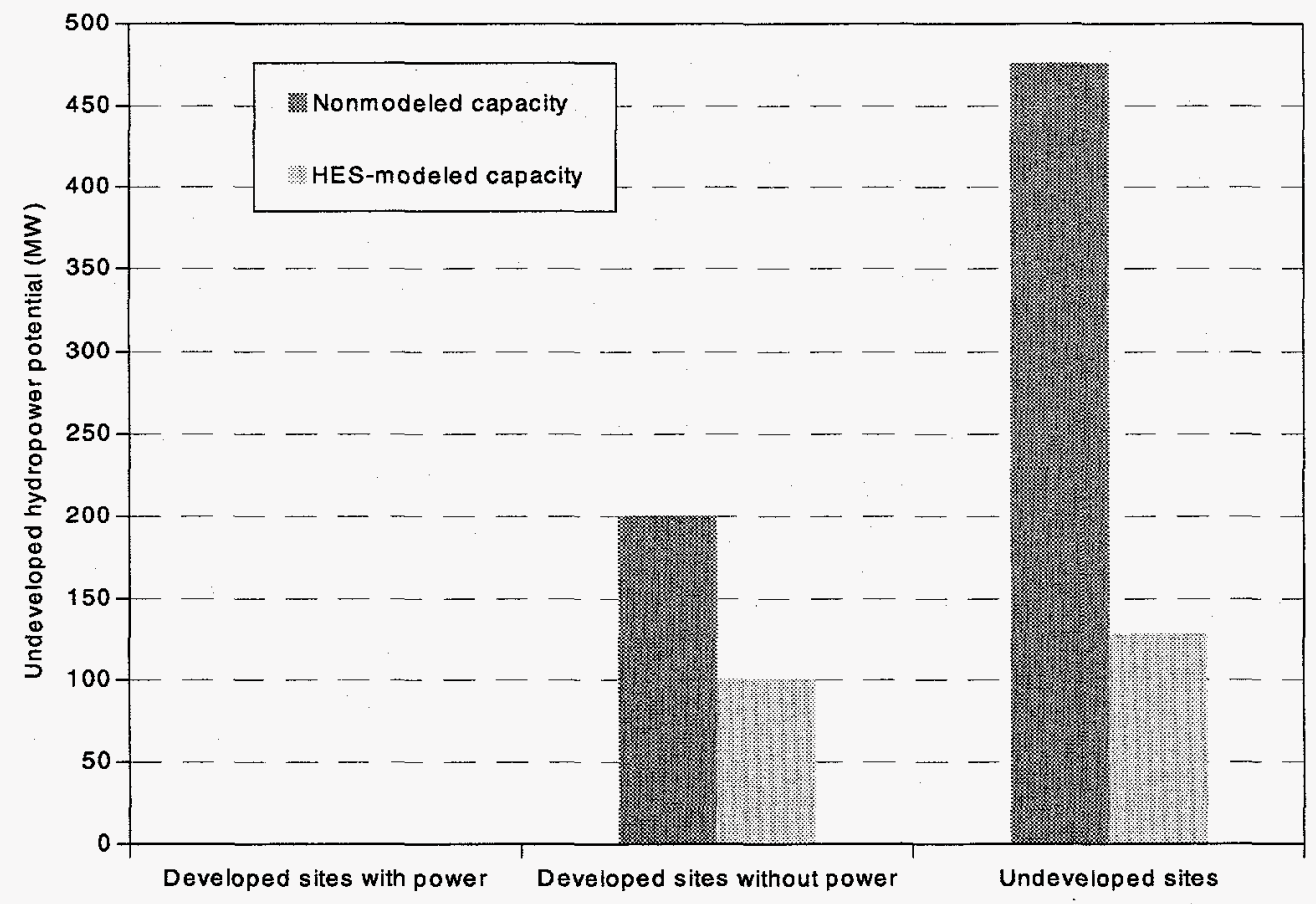

Figure 2. The nonmodeled and HES-modeled undeveloped hydropower potential. 


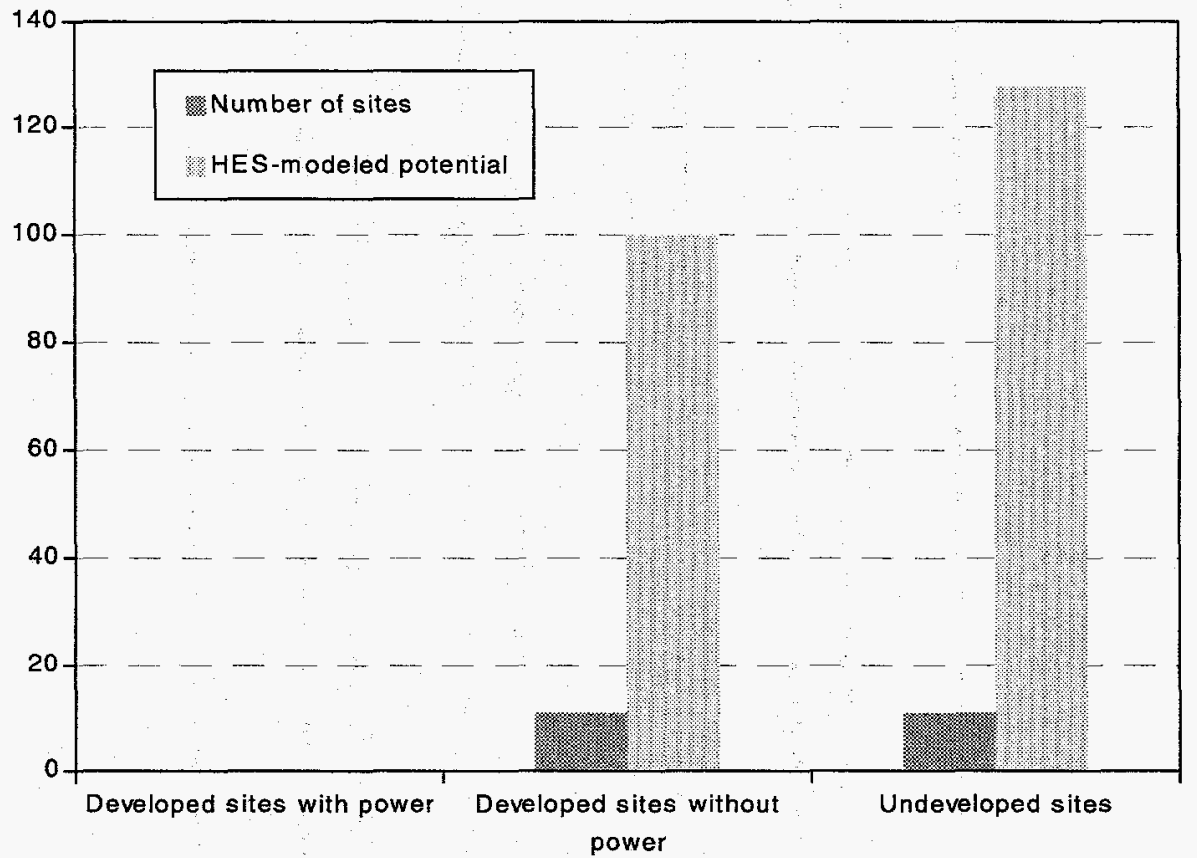

Figure 3. The number of sites with undeveloped hydropower potential and the total megawatts of HES-modeled undeveloped hydropower potential.

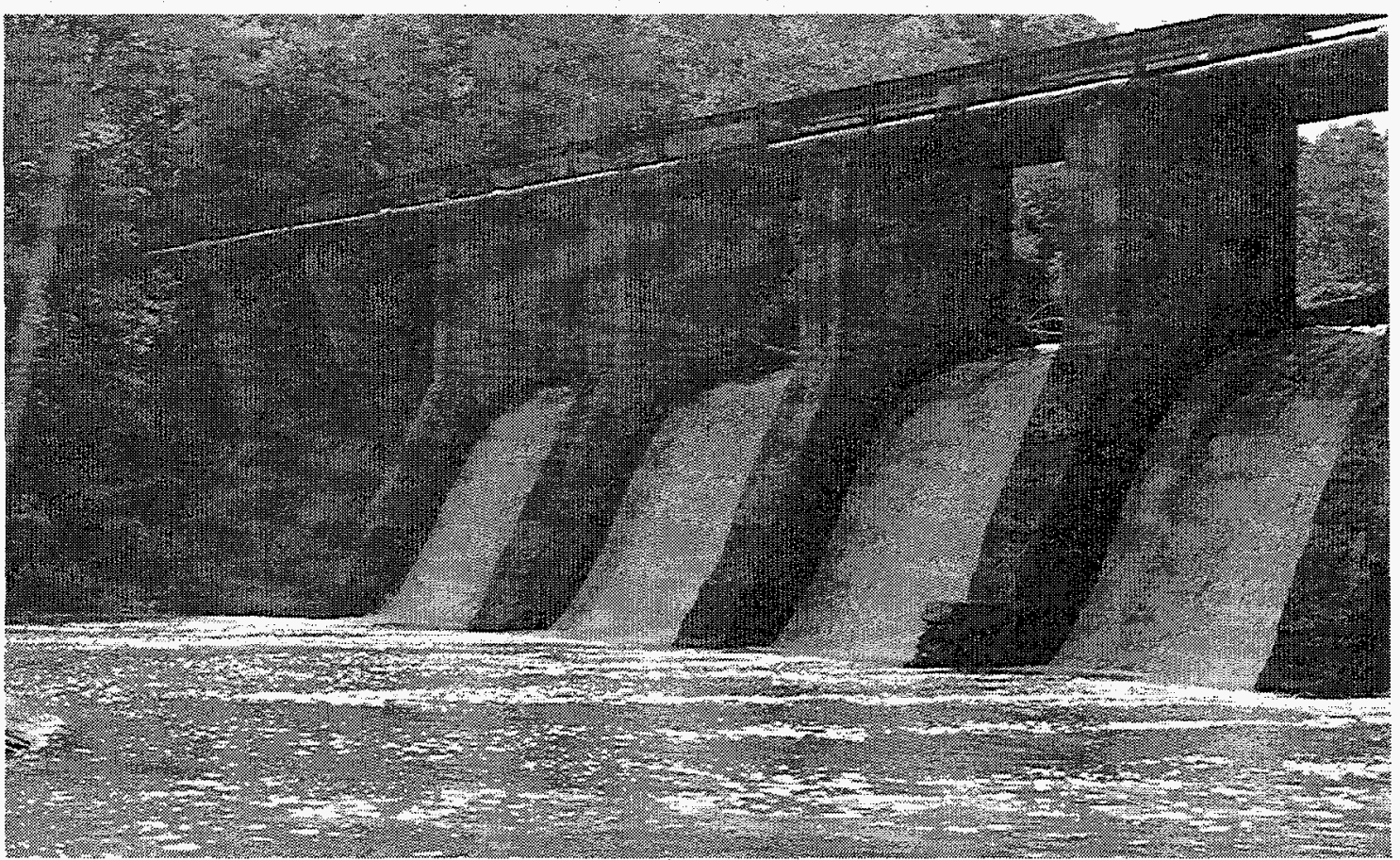

Figure 4. The Burgess Falls Dam in Putnam County, Tennessee. This dam produced power for Cookeville, Tennessee, before TVA was created in the 1930s. The dam is now in Burgess Falls State Natural Area. Concerns over its structural integrity may lead to its partial or total removal. 


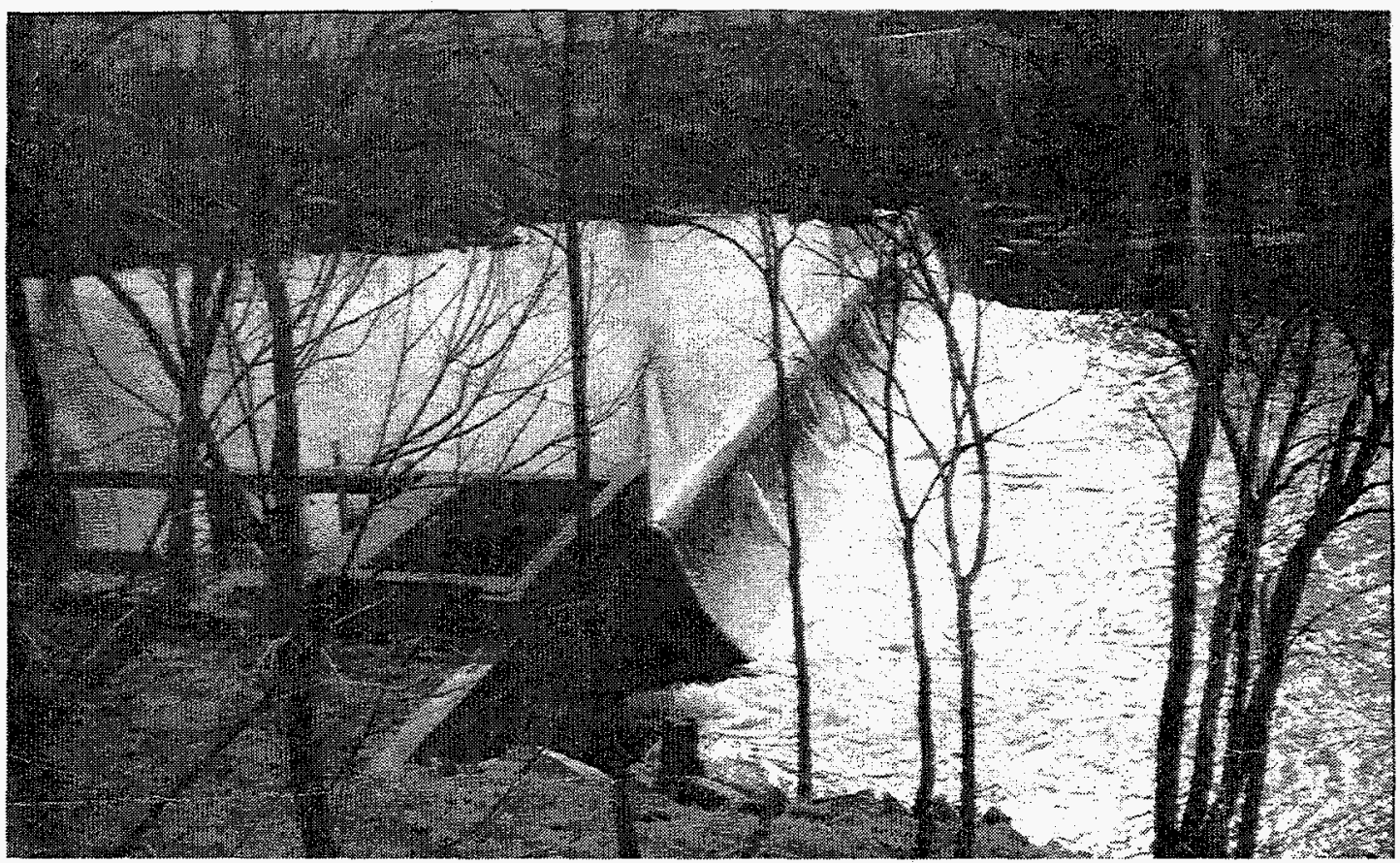

Figure 5. The Fairfield Mill Dam in Bedford County, Tennessee, was part of an old grist mill. A small power-generating station was added in the 1980s. The current owners gave up their license to produce power last year because it was uneconomical.

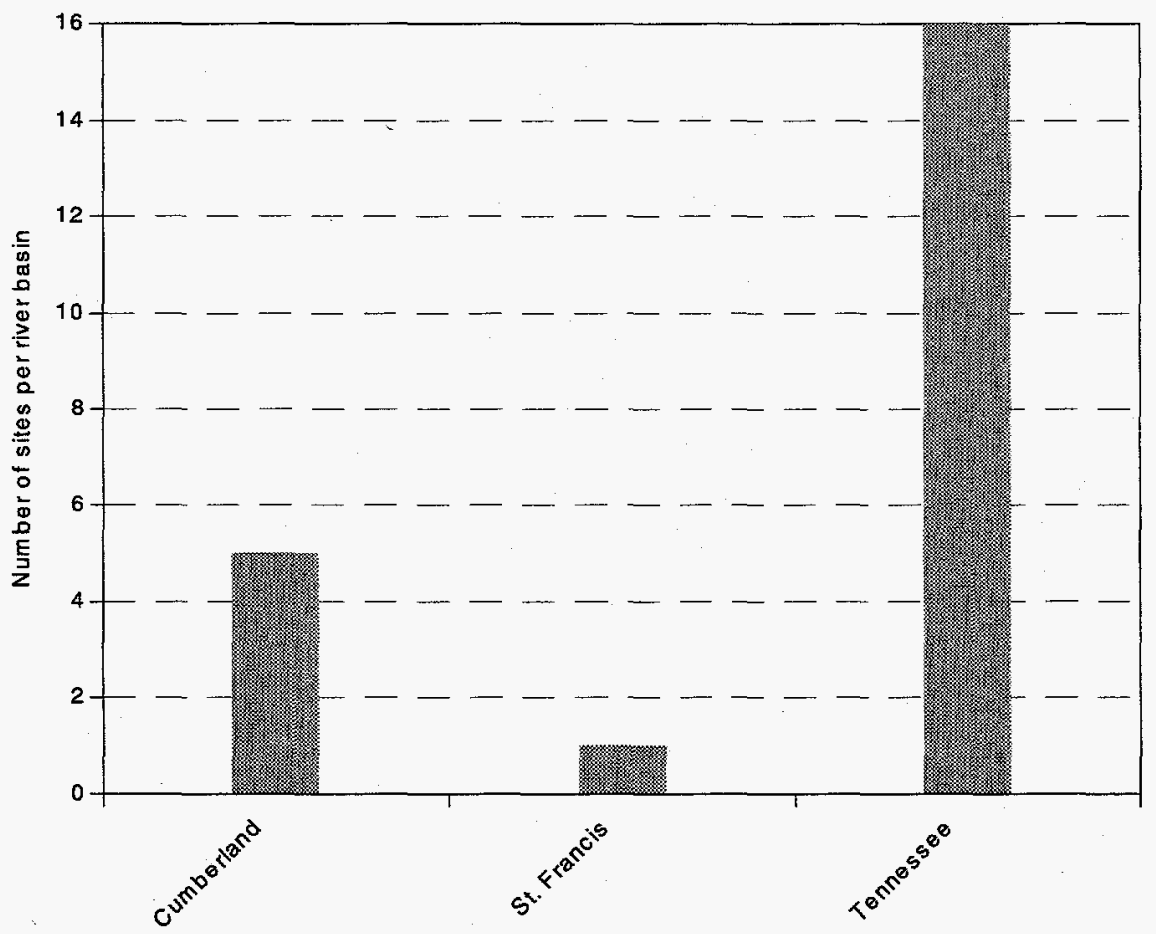

Figure 6. Number of sites with undeveloped hydropower potential in the Tennessee river basins. 


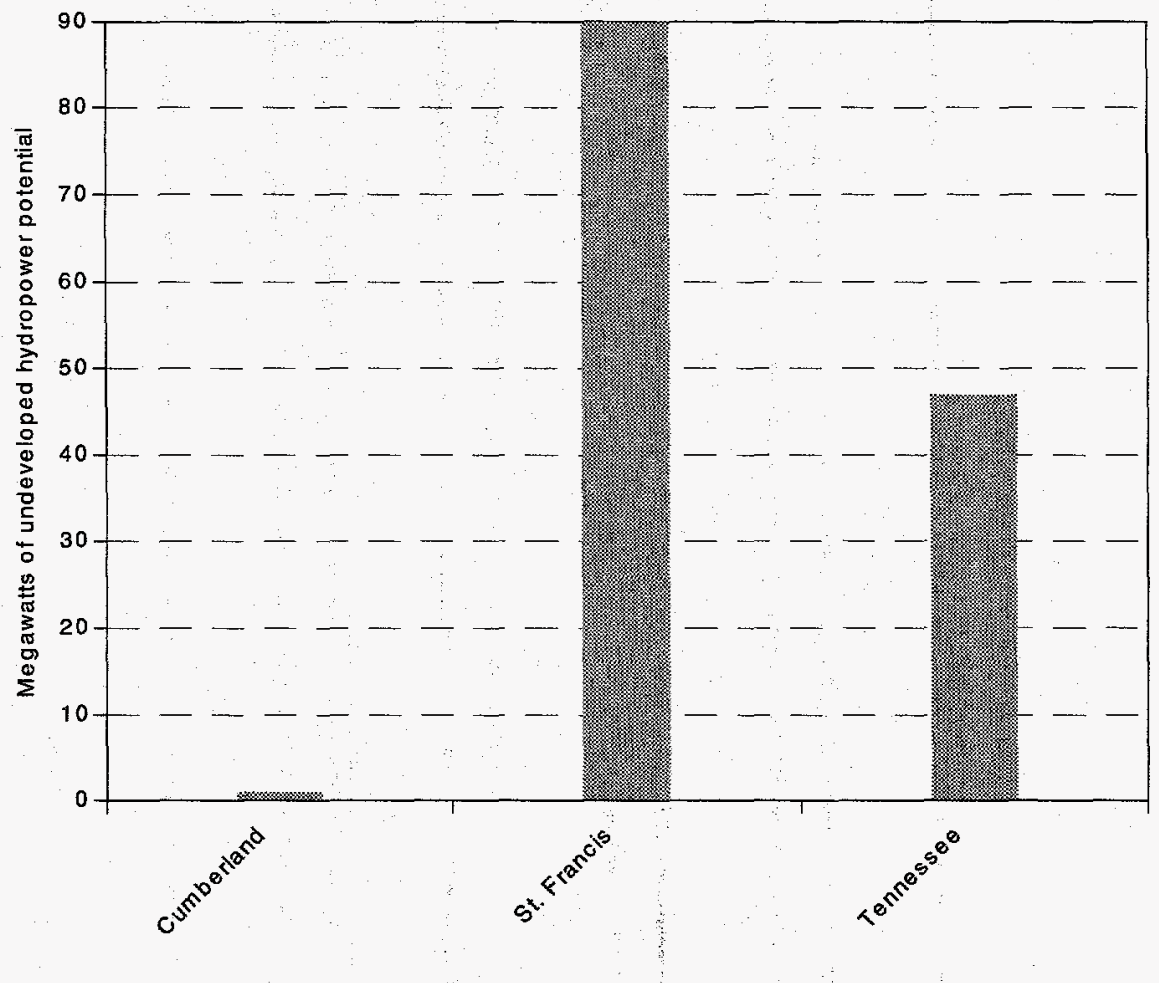

Figure 7. Megawatts of HES-modeled undeveloped hydropower potential in the Tennessee river basins.

\section{Detailed Results}

The appendices contain, in the form of HES-generated reports, detailed information about the undeveloped hydropower potential in Tennessee. The appendices contain the following information:

Appendix A summarizes the undeveloped hydropower potential by dam status groups. The number of sites, nonmodeled undeveloped hydropower potential, and HES-modeled undeveloped hydropower potential is provided based on the dam status.

Appendix B provides the hydropower resource assessment by river basin, which includes the project number, project name, stream name, dam status, nonmodeled undeveloped hydropower potential, and the HES-modeled undeveloped hydropower potential for each site. Subtotals are provided for each river basin.

Appendix $\mathbf{C}$ lists the project numbers, plant name, stream name, if a site is Federally owned, nonmodeled undeveloped hydropower potential, and HES-modeled undeveloped hydropower potential. The sites are grouped by dam status.

Appendix D contains a resource database list for the 22 sites in Tennessee. Information includes plant name, stream, state, county, river basin and owner names, project number, nameplate and HES-modeled undeveloped hydropower potential, the unit and plant types, dam status, latitude, longitude, and the environmental factors that the HES uses to determine the PESF.

\section{OBTAINING INDIVIDUAL STATE INFORMATION}

Additional copies of the hydropower resource assessment results for individual states are available and can be obtained by writing or calling the authors or the National Technical Information Service (NTIS).

Telephone Orders-(703) 487-4650. NTIS sales desk and customer services are 
available between 8:30 a.m. and 5:00 p.m., Eastern Standard Time.

Fax-(703) 321-8547. Customers may fax their orders to NTIS. These orders may be charged to a NTIS deposit account, American Express, VISA, or MasterCard.

Mail Orders-Mail orders should be sent to National Technical Information Service, Document Sales, 5285 Port Royal Road, Springfield, VA 22161. Call the sales desk for prices before placing an order.

E-mail-Customers may e-mail their requests to info@ntis.fedworld.gov.

Method of Payment-Customers may pay for reports (and other NTIS products and services) by (a) credit card (American Express, Visa or MasterCard); (b) check or money order on a United States bank payable to NTIS; (c) NTIS deposit account; or, (d) by asking to be billed (add \$7.50 per order), United States, Canada, and Mexico, only.

Handling Fee $-\mathrm{A} \$ 3.00$ handling fee per total order applies to orders from the United States, Canada, and Mexico. Handling charges do not apply to rush order service or pick-up orders.

Postage and Shipping-Orders are shipped first class mail, or equivalent, to addresses in the United States, Canada, and Mexico.

Order Turnaround Time-Orders for technical reports generally are shipped within 2 to 8 days of receipt. For faster service, NTIS offers rush order service.

Rush Order Service-Call 1-800-533-NTIS. In Virginia, Canada, and Mexico call (703) 487-4700 . For NTIS rush order service add $\$ 15.00$ per item. This guarantees that an order will be processed through NTIS within 24 hours of its receipt. These orders receive immediate, individual attention. The items ordered are delivered by first call mail. Call NTIS for information on rush order service for computer products.

For Help in Tracing an Order-Call (703) 487-4650 and request the customer service option.

\section{ADDITIONAL HYDROPOWER EVALUATION SOFTWARE INFORMATION}

Additional information concerning the HES can be obtained by contacting Ben Rinehart or Jim Francfort at the addresses provided below. Copies of the software and the User's Manual may also be obtained from these individuals.

Ben Rinehart, Project Manager

Idaho National Engineering and Environmental Laboratory

P.O. Box 1625, MS 3830

Idaho Falls, ID 83415-3830

(208) 526-1002

Jim Francfort

Idaho National Engineering and Environmental Laboratory

P.O. Box 1625, MS 3875

Idaho Falls, ID 83415-3875

(208) 526-6787

Information concerning the State of Tennessee's involvement with the resource assessment or about the identified sites may be obtained by contacting:

Lyle Bentley

Division of Water Supply

Tennessee Department of Environment and

Conservation

6th Floor, L\&C Tower

401 Church Street

Nashville, TN 37243-1549

(615) 532-0154. 


\section{REFERENCES}

Conner, A. M., J. E. Francfort, and B. N. Rinehart, 1996, Uniform Criteria for U.S. Hydropower Resource Assessment, Hydropower Evaluation Software Status Report-II, DOE/ID 10430.1, Idaho National Engineering Laboratory, Idaho Falls, Idaho.

Francfort, J. E., S. D. Matthews, and B. N. Rinehart, 1991, Hydropower Evaluation Software User's Manual, DOE/ID-10338, Idaho National Engineering Laboratory, Idaho Falls, Idaho. 
Appendix A

Summary Report 
DATE: $05 / 23 / 97$

\begin{tabular}{|c|c|c|c|c|}
\hline State & Category & $\begin{array}{l}\text { Number of } \\
\text { Projects }\end{array}$ & $\begin{array}{l}\text { Name Plate } \\
\text { Capacity }(\mathrm{KW})\end{array}$ & $\begin{array}{l}\text { HES Adjusted } \\
\text { Capacity }(\mathrm{KW})\end{array}$ \\
\hline \multirow[t]{4}{*}{$\mathrm{TN}$} & with Power & 0 & 0 & 0 \\
\hline & w/o Power & 11 & 20013 & 10016.50 \\
\hline & Undeveloped & 11 & 475908 & 127598.80 \\
\hline & STATE TOTAL & 22 & 495921 & 137615.30 \\
\hline \multirow[t]{3}{*}{ TOTALS } & With Power & 0 & 0 & 0 \\
\hline & W/O Power & 11 & 20013 & 10016.50 \\
\hline & Undeveloped & 11 & 475908 & 127598.80 \\
\hline GRAND : & TAL & 22 & 495921 & 137615.30 \\
\hline
\end{tabular}


Appendix B

River Basins Report 


\begin{tabular}{|c|c|c|c|c|c|}
\hline & HYDROPOWER RESOURCE & ASSESSM & ENT BY RIVER & BASIN & \\
\hline $\begin{array}{l}\text { FERC } \\
\text { Number }\end{array}$ & $\begin{array}{c}\text { Plant Name/ } \\
\text { Stream }\end{array}$ & $\begin{array}{l}\text { Dam } \\
\text { stat }\end{array}$ & $\begin{array}{l}\text { Name Plate } \\
\text { Rating }(\mathrm{KW})\end{array}$ & PESF & $\mathrm{PESF} * \mathrm{KW}$ \\
\hline $\begin{array}{l}* * \text { Rive } \\
09189\end{array}$ & $\begin{array}{l}\text { Basin CUMBERLAND RIVER BASIN } \\
\text { DALE HOLLOW } \\
\text { OBEY R }\end{array}$ & N & 98.00 & 0.10 & 9.80 \\
\hline 03617 & $\begin{array}{l}\text { WALTERHILI } \\
\text { E FK STONES R }\end{array}$ & wo & 508.00 & 0.50 & 254.00 \\
\hline 10590 & $\begin{array}{l}\text { BURGESS FALLS } \\
\text { FALIING WATER R }\end{array}$ & wo & 635.00 & 0.50 & 317.50 \\
\hline 07877 & $\begin{array}{l}\text { MCMINNVILLE } \\
\text { BARREN FK R, ROCKY R }\end{array}$ & wo & 280.00 & 0.50 & 140.00 \\
\hline 10589 & $\begin{array}{l}\text { OLD FACTORY DAM(SPARTA) } \\
\text { CALFKILLER R, CANEY FK }\end{array}$ & wo & 600.00 & 0.50 & 300.00 \\
\hline ** Subt & otal ** & & 2121.00 & & 1021.30 \\
\hline $\begin{array}{l}* * \text { Rive } \\
06994\end{array}$ & $\begin{array}{l}\text { I Basin ST FRANCIS RIVER BASIN } \\
\text { H M BESSIE } \\
\text { MISSISSIPPI R }\end{array}$ & N & 100000.00 & 0.90 & 90000.00 \\
\hline ** Subt & otal ** & & 100000.00 & & 90000.00 \\
\hline $\begin{array}{l}* * \text { Rive } \\
\text { TNO01 }\end{array}$ & $\begin{array}{l}\text { Basin TENNESSEE RIVER BASIN } \\
\text { COLUMBIA } \\
\text { DUCK R }\end{array}$ & $\mathrm{U}$ & 21780.00 & 0.10 & 2178.00 \\
\hline 09434 & $\begin{array}{l}\text { WARTBURG } \\
\text { CROOKED FK, EMORY } R\end{array}$ & $\mathbf{U}$ & 1400.00 & 0.10 & 140.00 \\
\hline TNO03 & $\begin{array}{l}\text { CUMBERLAND GAP } \\
\text { POWELL } \mathrm{R}\end{array}$ & $\mathrm{U}$ & 71400.00 & 0.10 & 7140.00 \\
\hline TNOO4 & $\begin{array}{l}\text { WAR RIDGE } \\
\text { CLINCH R }\end{array}$ & $\mathbf{U}$ & 113300.00 & 0.10 & 11330.00 \\
\hline 07624 & $\begin{array}{l}\text { RIVERDALE } \\
\text { FRENCH BROAD R }\end{array}$ & $\mathrm{U}$ & 40000.00 & 0.10 & 4000.00 \\
\hline TNO05 & $\begin{array}{l}\text { LONG CREEK } \\
\text { FRENCH BROAD R }\end{array}$ & $\mathrm{U}$ & 86100.00 & 0.10 & 8610.00 \\
\hline 07629 & $\begin{array}{l}\text { BEAVER CREEK } \\
\text { HOLSTON } R\end{array}$ & $\mathrm{U}$ & 30000.00 & 0.10 & 3000.00 \\
\hline
\end{tabular}




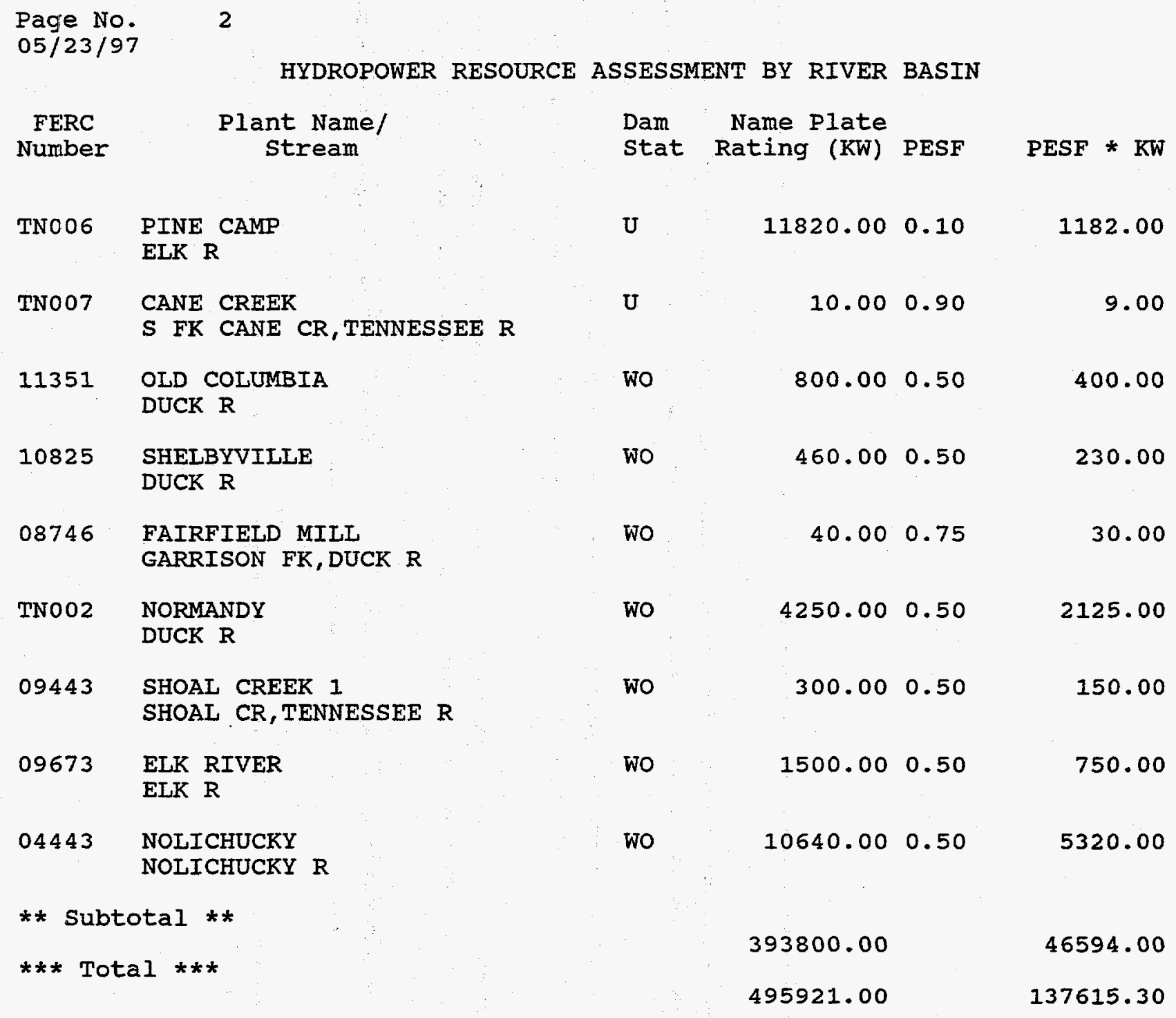


Appendix C

Tennessee Sites List 
HYDROPOWER RESOURCE ASSESSMENT BY FERC NUMBER

$$
\begin{gathered}
\text { Plant Name/ } \\
\text { Stream }
\end{gathered}
$$

** FERC Number 06994

H M BESSIE

MISSISSIPPI $R$

** Subtotal **

** FERC Number 07624

RIVERDALE

FRENCH BROAD R

** Subtotal **

** FERC Number 07629

BEAVER CREEK

HOLSTON $\mathbf{R}$

** Subtotal **

** FERC Number 09189

DALE HOLLOW

OBEY $R$

** Subtotal **

** FERC Number 09434 WARTBURG

CROOKED FK, EMORY $R$

** Subtotal **

** FERC Number TNOOI COLUMBIA

DUCK $R$

** Subtotal **

** FERC Number TN003 CUMBERLAND GAP POWELI R

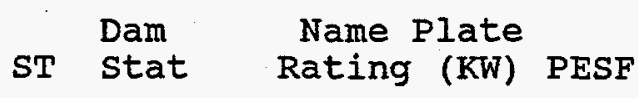

TN U

$1400.00 \quad 0.10$

140.00

1400.00

140.00

TN U

$21780.00 \quad 0.10$

2178.00

21780.00

2178.00

TN U

$71400.00 \quad 0.10$

7140.00 
Page No.

$05 / 23 / 97$

2

HYDROPOWER RESOURCE ASSESSMENT BY FERC NUMBER

Plant Name/

stream

** Subtotal **

** FERC Number TNOO4

WAR RIDGE

CLINCH R

** Subtotal **

** JPERC Number TNO05

LONG CREEK

FRENCH BROAD R

** Subtotal **

** FERC Number TN006

PINE CAMP

ELK R

** Subtotal **

** FERC Number TNO07

CANE CREEK

$S$ FK CANE CR, TENNESSEE $R$

** Subtotal **

** FERC Number 03617

WALTERHILL,

E FK STONES $R$

** Subtotal **

** FERC Number 04443

NOIICHUCKY

NOLICHUCKY $R$

** Subtotal ** $\begin{array}{llll} & \text { Dam Name Plate } & \\ \text { ST Stat } & \text { Rating (KW) PESF } & \text { PESF * KW }\end{array}$

71400.00

7140.00

TN U

$113300.00 \quad 0.10$

11330.00

113300.00

11330.00

TN U

86100.00 .0 .10

8610.00

86100.00

8610.00

TN U

$11820.00 \quad 0.10$

1182.00

11820.00

1182.00

TN U

$10.00 \quad 0.90$

9.00

10.00

9.00

TN Wo

$508.00 \quad 0.50$

254.00

508.00

254.00

TN WO

$10640.00 \quad 0.50$

5320.00

10640.00

5320.00 
Page No. $\quad 3$

$05 / 23 / 97$

HYDROPOWER RESOURCE ASSESSMENT BY FERC NUMBER

Plant Name/ stream

** FERC Number 07877 MCMINNVILLE

BARREN FK R, ROCKY R

** Subtotal **

** FERC Number 08746

FAIRFIELD MILL

GARRISON FK, DUCK R

** Subtotal **

** FERC Number 09443

SHOAL CREEK 1

SHOAI CR, TENNESSEE $R$

** Subtotal **

** FERC Number 09673

EIK RIVER

ELK R

** Subtotal **

** FERC Number 10589

OLD FACTORY DAM (SPARTA)

CALFKILIER R, CANEY FK

** Subtotal **

** FERC Number 10590

BURGESS FAILS

FALIING WATER $R$

** Subtotal **

** FERC Number 10825

SHELBYVILLE

DUCK $\mathbf{R}$ $\begin{array}{lll}\text { Dam } & \text { Name Plate } & \\ \text { ST Stat } & \text { Rating (KW) PESF } & \text { PESF * KW }\end{array}$

TN WO

$280.00 \quad 0.50$

140.00

280.00

140.00

TN WO

$40.00 \quad 0.75$

30.00

40.00

30.00

TN WO

$300.00 \quad 0.50$

150.00

300.00

150.00

TN WO

1500.00

0.50

750.00

1500.00

750.00

TN Wo

$600.00 \quad 0.50$

300.00

600.00

300.00

TN WO

$635.00 \quad 0.50$

317.50

635.00

317.50

TN WO

$460.00 \quad 0.50$

230.00 
Page No. 05/23/97

4

HYDROPOWER RESOURCE ASSESSMENT BY FERC NUMBER

$$
\begin{aligned}
& \text { Plant Name/ } \\
& \text { Stream }
\end{aligned}
$$

** Subtotal **

** FERC Number 11351

OLD COLUMBIA

DUCK $R$

** Subtotal **

** FERC Number TNOO2 NORMANDY

DUCK $R$

** Subtotal **

*** Total ***

$\begin{array}{ll}\text { Dam } & \text { Name Plate } \\ \text { ST Stat } & \text { Rating (KW) PESF }\end{array}$

$$
460.00
$$

TN WO

800.00

TN WO

$4250.00 \quad 0.50$

4250.00

495921.00
PESF * KW

230.00

400.00

400.00

2125.00

2125.00

137615.30 
Appendix D

Individual Resource Database List 
DATE: $05 / 23 / 97$

PAGE NO: 1

FERC

Number

Plant Name

Stream

State

03617

WALTERHILL

E FK STONES R

Name

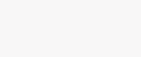

County Name

River Basin

RUTHERFORD

CUMBERIAND RIVER BASIN

Class Owner Name

M CITY OF MURFREESBORO

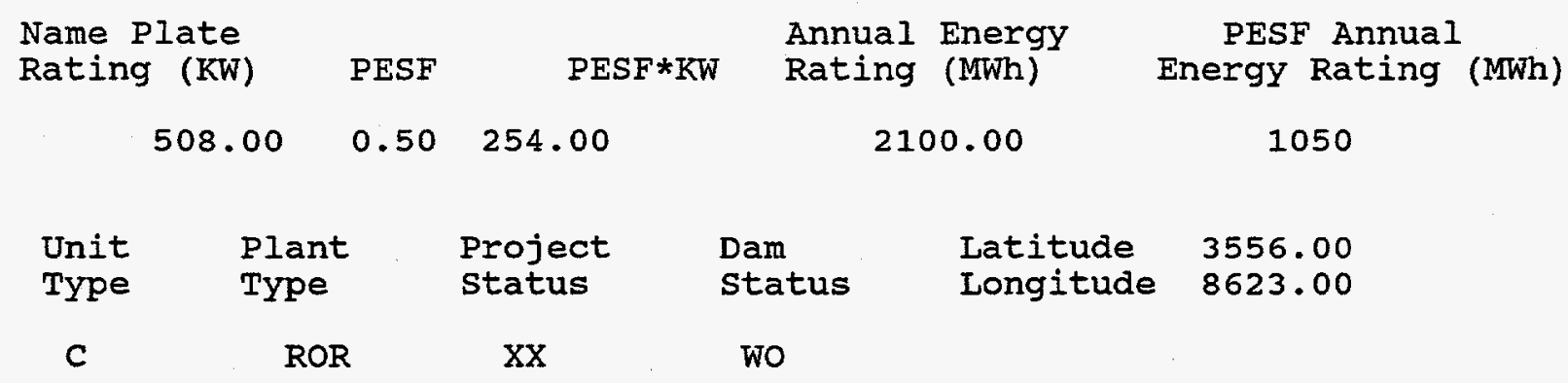

Factor

Wild/Scenic Protection

wild/Scenic Tributary or Upstream/Downstream wild/Scenic Location Cultural value Fish Presence Value Geologic Value Historic Value Other Value Recreation value Scenic Value
Exist Prob

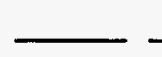

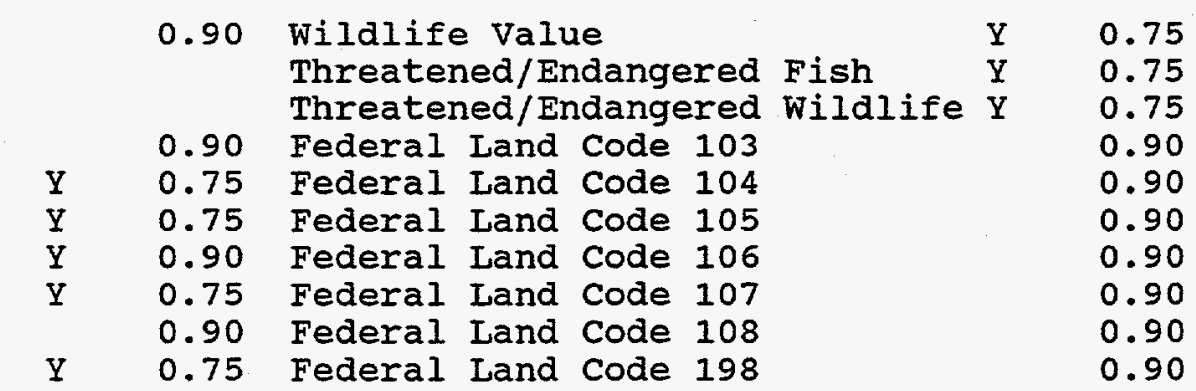


DATE: $05 / 23 / 97$

PAGE NO: 2

FERC

Number

Plant Name

stream

State

Name

04443 NOLICHUCKY

NOLICHUCKY $R$

TN

County Name

GREENE
River Basin

TENNESSEE RIVER BASIN

class Owner Name

F TENNESSEE VALLEY AUTHORITY

Name Plate

Rating (KW)

PESF

Annual Energy

10640.00

$0.50 \quad 5320.00$

50000.00

PESF Annual Energy Rating (MWh)

$\begin{array}{cccccc}\text { Unit } & \text { Plant } & \text { Project } & \text { Dam } & \text { Iatitude } & 3604.00 \\ \text { Type } & \text { Type } & \text { Status } & \text { Status } & \text { Longitude } & \mathbf{8 2 5 2 . 0 0} \\ \text { C } & \text { RES } & \text { XX } & \text { wo } & \end{array}$

Factor

Exist Prob

Factor

Exist Prob

Wild/Scenic Protection

wild/Scenic Tributary or Upstream/Downstream

wild/Scenic Location Cultural value

Fish Presence Value

Geologic Value

Historic Value

Other Value

Recreation Value

Scenic Value

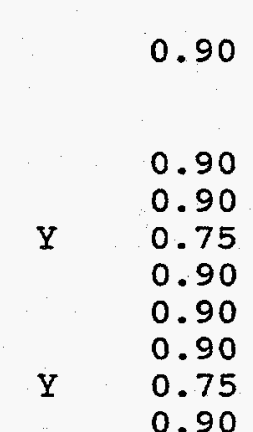

wildlife Value

Threatened/Endangered Fish

Threatened/Endangered Wildlife

Federal Land Code 103

Federal Land code 104

Federal Land code 105

Federal Land Code 106

Federal Land Code 107

Federal Land code 108

Federal Land Code 198
0.90

0.90

0.90

0.90

0.90

0.90

0.90

0.90

$\mathrm{Y} \quad 0.75$

0.90 
DATE: $05 / 23 / 97$

PAGE NO: 3

FERC

Number

Plant Name

stream

state

06994

H M BESSIE

MISSISSIPPI $\mathbf{R}$

Name

\section{(16994}

H BESSIP

county Name

River Basin

LAKE

ST FRANCIS RIVER BASIN

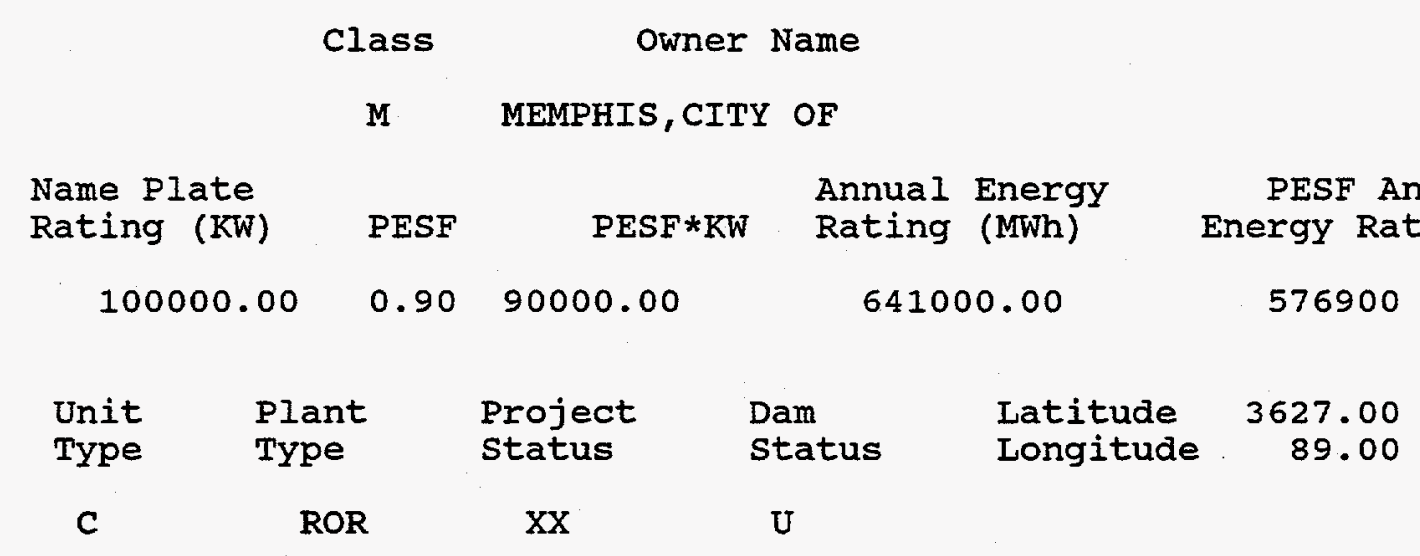

Factor

Wild/Scenic Protection

wild/scenic Tributary or

Upstream/Downstream

wild/Scenic Location

cultural Value

Fish Presence Value

Geologic Value

Historic Value

other Value

Recreation value

Scenic Value
Exist Prob

0.90

Wildlife Value Threatened/Endangered Fish Threatened/Endangered Wildlife

0.90 Federal Land code 103

0.90 Federal Land Code 104

0.90 Federal Land code 105

0.90 Federal Land code 106

0.90 Federal Land code 107

0.90 Federal Land code 108

0.90 Federal Land Code 198

0.90
TN

\begin{tabular}{ll} 
Exist & Prob \\
\cline { 2 - 2 } & \\
& 0.90 \\
& 0.90 \\
0.90 \\
0.90 \\
0.90 \\
0.90 \\
0.90 \\
0.90 \\
0.90 \\
0.90
\end{tabular}


FERC

Number

Plant Name

07624 RIVERDALE

county Name

KNOX

\section{Class}

$R$ INDEPENDENCE ELEC CORP

Name Plate

Rating ( $\mathrm{KW})$

PESF

PESF*KW

Annual Energy

40000.00

0.104000 .00

Plant

Type

ROR
Project status

XX
River Basin

TENNESSEE RIVER BASIN
FRENCH BROAD $\mathbf{R}$

133000.00

U
State

Name

TN

PESF Annual Energy Rating (MWh)

13300

$\begin{array}{lll}\text { Dam } & \text { Latitude } & 3556.00 \\ \text { Status } & \text { Longitude } & 8345.00\end{array}$

Factor

Wild/Scenic Protection wild/Scenic Tributary or Upstream/Downstream wild/scenic Location Cultural value

Fish Presence Value

Geologic Value

Historic Value

other Value

Recreation Value

Scenic Value
Exist Prob

Factor

Exist Prob

$\begin{array}{ll}Y & 0.10 \\ & \\ & \\ Y & 0.90 \\ Y & 0.50 \\ Y & 0.25 \\ Y & 0.50 \\ Y & 0.50 \\ Y & 0.90 \\ Y & 0.25\end{array}$

Wildife Value Threatened/Endangered Fish Threatened/Endangered Wildife $Y$

$Y$

0.25 Federal Iand Code 103 Federal Land Code 104

Federal Land Code 105

Federal Land Code 106

Federal Land Code 107

Federal Land Code 108

Federal Land code 198
0.50

0.50

0.90

0.90

0.90

0.90

0.90

0.90

0.90 
FERC

Number

07629
Plant Name

BEAVER CREEK

County Name

GRAINGER

Class
Stream

HOLSTON $\mathbf{R}$ state

Name

TN

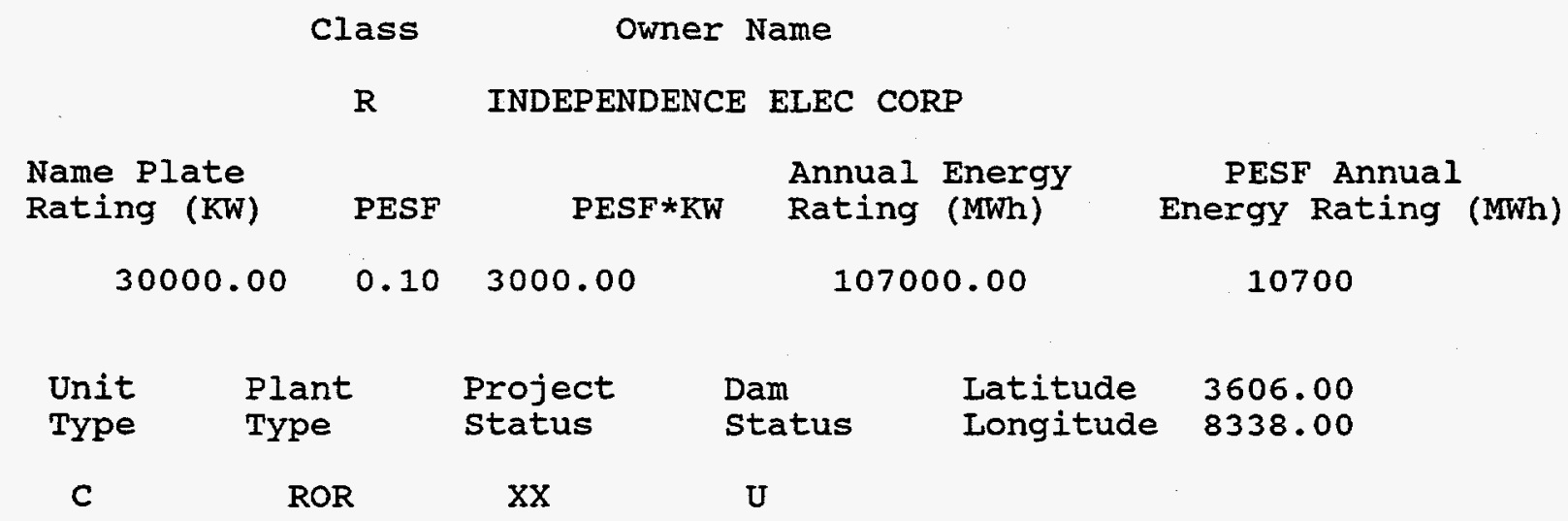

Factor

Wild/Scenic Protection wild/Scenic Tributary or Upstream/Downstream wild/Scenic Location Cultural Value Fish Presence Value Geologic Value Historic Value other Value Recreation value Scenic Value
Exist Prob

\section{$\mathrm{Y}$} 0.10

0.10

0.90

0.50

0.25

0.50

0.50

0.90

$\mathrm{Y} \quad 0.25$

$\mathrm{Y} \quad 0.50$
River Basin

TENNESSEE RIVER BASIN

Exist Prob

Wildlife Value Threatened/Endangered Fish

$Y$ 0.25

0.50 Threatened/Endangered Wildlife $Y \quad 0.50$ Federal Land Code 103

0.90

Federal Land Code 104

0.90

Federal Land Code 105

0.90

Federal Land Code 106

0.90

Federal Land Code 107

0.90

Federal Land Code 108

0.90

Federal Land Code 198

0.90 
DATE: $05 / 23 / 97$

PAGE NO: 6

FERC

Number

07877
Plant Name

MCMINNVILLE

County Name

WARREN
State

Name

TN
BARREN FK R, ROCKY R

River Basin

CUMBERLAND RIVER BASIN

Class Owner Name

R CITY OF MCMINNVILLE

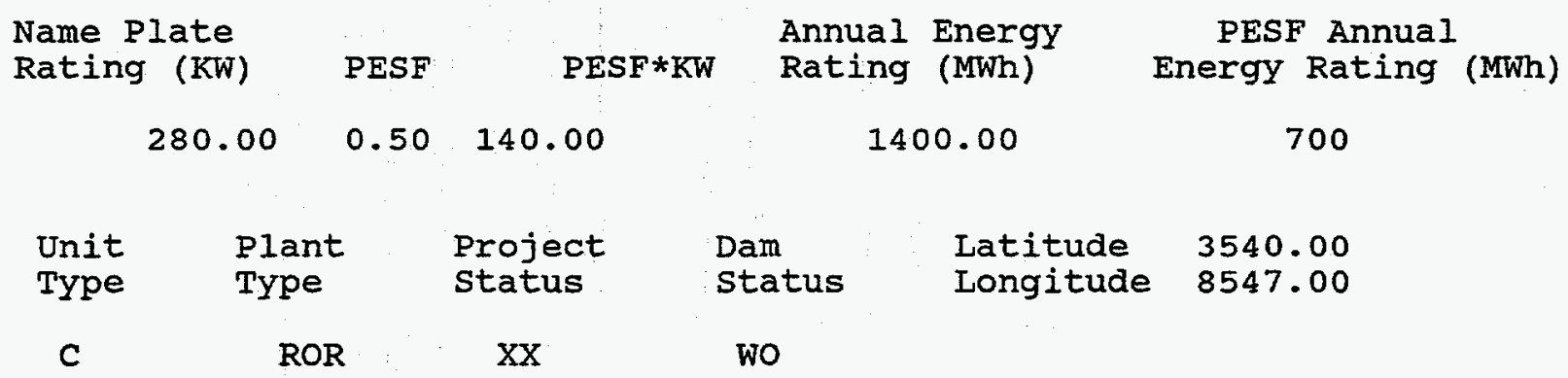

Factor

Exist Prob

wild/Scenic Protection

wild/Scenic Tributary or Upstream/Downstream wild/Scenic Location Cultural Value

Fish Presence Value

Geologic Value

Historic Value other value

Recreation Value

Scenic Value
Factor

Exist Prob

0.90

Wildife Value

Threatened/Endangered Fish

Threatened/Endangered Wildlife Y

0.90 Federal Land Code 103

0.90 Federal Land Code 104

Federal Land Code 105

0.90 Federal Land Code 106
0.90 Federal Land Code 107

0.90 Federal Land code 108

0.90 Federal Iuand Code 198

0.90
0.90

0.90

0.75

0.90

0.90

0.90

0.90

0.90

0.90

0.90 
DATE: $05 / 23 / 97$

PAGE NO: 7

FERC

Number

Plant Name

stream

State

08746

FAIRFIELD MILL

GARRISON FK, DUCK R

Name

county Name

River Basin

BEDFORD

TENNESSEE RIVER BASIN

class Owner Name

R HICKERSON, LOGAN

Name Plate

PESF PESF*KW

Annual Energy

Rating (MWh)

150.00

Latitude $\quad 3533.00$

Unit Plant Project

Type Type status

C

ROR

$\mathrm{XX}$

Dam
Status

WO
PESF Annual Energy Rating (MWh) 112.50
Factor

Wild/Scenic Protection

Wild/Scenic Tributary or Upstream/Downstream wild/Scenic Location Cultural Value Fish Presence Value Geologic Value Historic Value other value Recreation Value Scenic Value
Exist Prob

0.90

Wildife Value Threatened/Endangered Fish Threatened/Endangered Wildlife

0.90

0.90

0.90

0.90

0.90

0.90

0.90

Federal Land Code 103

Federal Land Code 104

Federal Land Code 105

Federal Land Code 106

Federal Land Code 107

Federal Land Code 108

Federal Land Code 198
TN

0.90

Exist Prob

0.90

0.75

0.90

0.90

0.90

0.90

0.90

0.90

0.90

0.90 
DATE: $05 / 23 / 97$

FERC Number 09189
DALE HOLLOW

county Name

CLAY
PAGE NO: 8

State

Name

OBEY $R$

TN

River Basin

CUMBERLAND RIVER BASIN

class Owner Name

$R$ JDJ ENERGY CO

Name Plate Rating (KW)
Annual Energy Rating (MWh)
PESF Annual Energy Rating (MWh)

$$
98.00
$$

$$
0.10 \quad 9.80
$$

772.00

77.20

$\begin{array}{ccccc}\text { Unit } & \text { Plant } & \text { Project } & \text { Dam } & \text { Latitude } \\ \text { TYpe } & \text { Type } & \text { Status } & \text { Status } & \text { Longitude } 852.00 \\ \text { C } & \text { ROR } & \mathrm{XX} & \mathrm{U} & \end{array}$

Factor

Wild/Scenic Protection

Wild/Scenic Tributary or Upstream/ Downstream wild/Scenic Location Cultural value Fish Presence Value Geologic Value Historic Value other value Recreation Value Scenic Value
Exist Prob

\section{$\mathbf{Y}$}

$$
0.10
$$

Factor

Exist Prob

0.10

Wildlife Value Threatened/Endangered Fish Threatened/Endangered Wildlife, $Y$

0.90 Federal Land Code 103

0.90 Federal Land Code 104

Y $\quad 0.25$ Federal Land Code 105

$Y \quad 0.50$ Federal Land Code 106

0.90 Federal Land Code 107

0.90 Federal Land Code 108

$\mathrm{Y} \quad 0.25$

Federal Land Code 198
Exist Prob

0.25

0.90

0.50

0.90

0.90

0.90

0.90

0.90

$\mathrm{Y} \quad 0.50$
0.90 
FERC

Number

Plant Name

09434

WARTBURG

County Name

MORGAN

Class

Owner Name

R CAREX HYDRO

Name Plate Rating ( $\mathrm{KW})$

PESF

$\mathrm{PESF} * \mathrm{KW}$

Annual Energy Rating (MWh)

7000.00

$0.10 \quad 140.00$

1400.00

Project

Unit
Type

Plant

Type

C

ROR

Factor

wild/Scenic Protection wild/Scenic Tributary or

Upstream/Downstream

wild/scenic Location

Cultural value

Fish Presence Value

Geologic Value

Historic Value

other value

Recreation Value

Scenic Value

\begin{abstract}
status
\end{abstract}
$\mathrm{XX}$

Exist Prob

$\mathbf{Y}$

0.10

Wildife Value Threatened/Endangered Fish

Threatened/Endangered Wildlife

Exist Prob

$\begin{array}{ll}0.90 & \text { Federal Land Code } 103 \\ 0.90 & \text { Federal Land code } 104\end{array}$

$Y \quad 0.25$ Federal Land code 105

$\mathrm{Y} \quad 0.50$ Federal Land Code 106

0.90 Federal Land code 107

0.90 Federal Land code 108

Y 0.25 Federal Land code 198

$\begin{array}{ll}Y & 0.25 \\ Y & 0.50\end{array}$
3604.00

8435.00
State

Name

TN
PESF Annual

Energy Rating (MWh)

700 
DATE: $05 / 23 / 97$

FERC

Number

09443
Plant Name

SHOAI CREEK 1

county Name

LAWRENCE

\section{stream}

SHOAI CR, TENNESSEE $R$

River Basin

TENNESSEE RIVER BASIN
State

Name

TN

$$
\begin{array}{cc}
\text { Class } & \text { Owner Name } \\
\text { M } & \text { CITY OF IAAWRENCEBURG }
\end{array}
$$

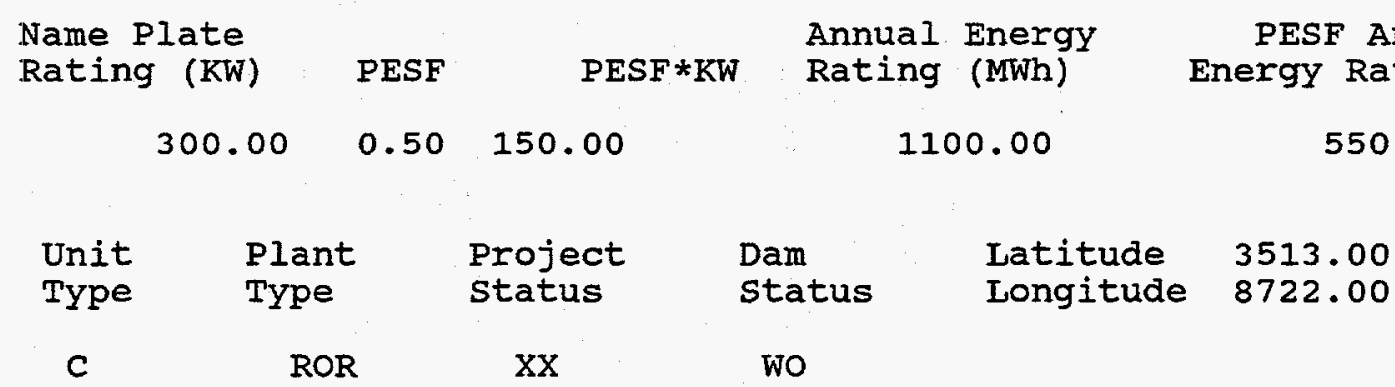

Factor

wild/Scenic Protection

wild/Scenic Tributary or

Upstream/Downstream

wild/scenic Location

Cultural Value

Fish Presence Value

Geologic Value

Historic Value

other Value

Recreation value

scenic Value
Exist Prob

0.90

Wildlife Value

Threatened/Endangered Fish

Threatened/Endangered wildlife

0.90 Federal Land code 103

0.90 Federal Land Code 104

Y 0.75 Federal Land code 105

0.90 Federal Land Code 106

0.90 Federal Land code 107

0.90 Federal Land Code 108

$Y \quad 0.75$ Federal Land code 198

$\begin{array}{ll}Y & 0.75 \\ Y & 0.90\end{array}$
Exist Prob

0.90

0.90

0.90

0.90

0.90

0.90

0.90

0.90

0.90

0.90 
FERC

Number

09673

ELK RIVER

county Name

FRANKIIN
Plant Name

ELK R
State

Name

TN

\section{class Owner Name}

F ARNOLD ENGINEERING DEVELOPMENT CENT

\begin{tabular}{|c|c|c|c|c|c|c|}
\hline \multicolumn{2}{|c|}{ Name Plate } & PESF & PESF $* K W$ & \multicolumn{2}{|c|}{$\begin{array}{l}\text { Annual Energy } \\
\text { Rating (MWh) }\end{array}$} & $\begin{array}{l}\text { PESF Annual } \\
\text { Inergy Rating (MWh) }\end{array}$ \\
\hline & .00 & 0.50 & 750.00 & \multicolumn{2}{|c|}{6927.00} & 3463.50 \\
\hline $\begin{array}{l}\text { Unit } \\
\text { Type }\end{array}$ & $\begin{array}{l}\text { Pla } \\
\text { TyY }\end{array}$ & & $\begin{array}{l}\text { Project } \\
\text { status }\end{array}$ & $\begin{array}{l}\text { Dam } \\
\text { status }\end{array}$ & $\begin{array}{l}\text { Latitude } \\
\text { Longitude }\end{array}$ & $\begin{array}{l}3518.00 \\
8606.00\end{array}$ \\
\hline$c$ & & & $\mathrm{XX}$ & wo & & \\
\hline
\end{tabular}

Factor

Wild/Scenic Protection wild/Scenic Tributary or Upstream/Downstream wild/Scenic Location Cultural Value Fish Presence Value Geologic Value Historic Value other Value Recreation Value Scenic Value
Exist Prob

0.90

Wildlife value Threatened/Endangered Fish Threatened/Endangered Wildlife $Y$

0.90 Federal Land Code 103

$\begin{array}{lll}Y & 0.75 & \text { Federal Land Code } 104 \\ \mathrm{Y} & 0.75 & \text { Federal Land Code } 105\end{array}$

0.90

Federal Land Code 106

$Y \quad 0.75$

0.90

$\mathrm{Y} \quad 0.75$

Federal Land Code 107

Federal Land Code 108

Federal Land Code 198
Exist Prob

$\mathrm{Y}$

0.75

0.90

0.75

0.90

0.90

0.90

0.90

0.90

0.90

0.90 
DATE: $05 / 23 / 97$

PAGE NO: 12

FERC

Number

10589
Plant Name

OLD FACTORY DAM (SPARTA)

County Name

WHITE
State

Name

CALFKILLER R, CANEY FK

TN

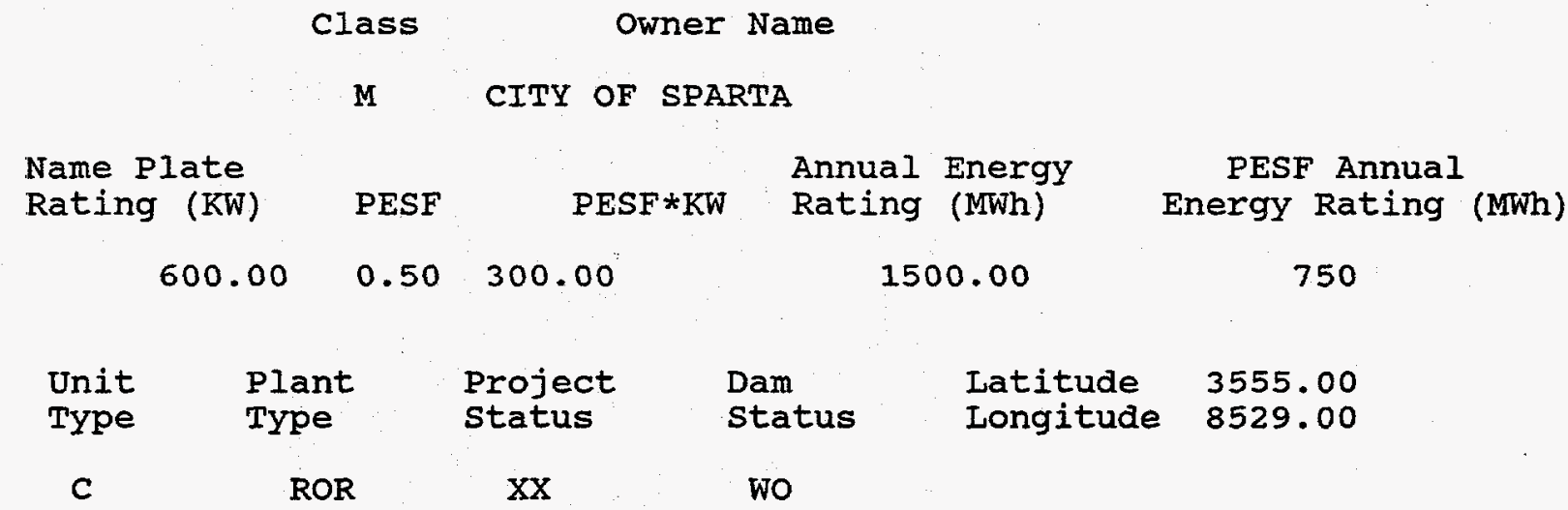

Factor

Wild/scenic Protection wild/Scenic Tributary or Upstream/Downstream Wild/Scenic Location Cultural Value Fish Presence Value Geologic Value Historic Value other value Recreation Value Scenic Value
Exist Prob

0.90

Wildlife Value Threatened/Endangered Fish Threatened/Endangered Wildlife $Y$

Exist Prob

0.90 Federal Land code 103
0.90 Federal Land code 104

0.75 Federal Land code 105

0.90 Federal Land code 106

0.90 Federal Land code 107

0.90 Federal Iand code 108

$\mathrm{Y} \quad 0.75$ Federal Land Code 198
Y

0.75

0.90

0.75

0.90

0.90

0.90

0.90

0.90

0.90

0.90
Exist Prob 
R E S O U R C E

DATE: $05 / 23 / 97$
D A T A B A S E I I S T I N G

PAGE NO: 13

\section{FERC}

Number

Plant Name

10590

BURGESS FALLS

County Name

PUTNAM

stream

FALLING WATER $R$

State

Name

TN

class Owner Name

M STATE OF TENNESSEE

\begin{tabular}{|c|c|c|c|c|c|c|}
\hline \multicolumn{2}{|c|}{ Name Plate } & PESF & PESF*KW & \multicolumn{2}{|c|}{$\begin{array}{l}\text { Annual Energy } \\
\text { Rating (MWh) }\end{array}$} & $\begin{array}{l}\text { PESF Annual } \\
\text { inergy Rating (MWh) }\end{array}$ \\
\hline & 635.00 & 0.50 & 317.50 & \multicolumn{2}{|c|}{1500.00} & 750 \\
\hline $\begin{array}{l}\text { Unit } \\
\text { Type }\end{array}$ & $\begin{array}{l}\text { Ple } \\
\text { Tyt }\end{array}$ & & $\begin{array}{l}\text { Project } \\
\text { Status }\end{array}$ & $\begin{array}{l}\text { Dam } \\
\text { Status }\end{array}$ & $\begin{array}{l}\text { Latitude } \\
\text { Iongitude }\end{array}$ & $\begin{array}{l}3602.00 \\
8535.00\end{array}$ \\
\hline C & \multicolumn{2}{|c|}{ ROR } & $\mathrm{XX}$ & \multicolumn{2}{|l|}{ wo } & \\
\hline
\end{tabular}

Factor

Wild/Scenic Protection

wild/Scenic Tributary or

Upstream/Downstream

wild/scenic Location

Cultural Value

Fish Presence Value

Geologic Value

Historic Value

other Value

Recreation value

Scenic Value
Exist Prob

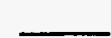

0.90

Wildlife Value

Threatened/Endangered Fish

Threatened/Endangered Wildlife

0.90 Federal Land Code 103

0.90 Federal Iand Code 104

$Y \quad 0.75$ Federal Land code 105

Y 0.90 Federal Land code 106

0.90 Federal Land code 107

0.90 Federal Land Code 108

$Y \quad 0.75$ Federal Land code 198

$\mathrm{Y} \quad 0.90$
Exist Prob

0.75

0.75

0.90

0.90

0.90

0.90

0.90

0.90

0.90

0.90 
DATE: $05 / 23 / 97$

PAGE NO: 14

FERC

Number

10825
Plant Name

SHELBYVILLE

county Name

BEDFORD
State

Name

DUCK R

TN

River Basin

TENNESSEE RIVER BASIN

Name Plate Rating (KW)

460.00

\section{Class}

M

CITY

Owner Name

F SHELBYVILLE

$\begin{array}{ll}\text { PESF } & \text { Annual Energy } \\ \text { RESF*KW } & \text { Rating (MWh) }\end{array}$

PESF Annual Energy Rating (MWh) $0.50 \quad 230.00$ 1800.00 900

$\begin{array}{cccll}\text { Unit } & \text { Plant } & \text { Project } & \text { Dam } & \text { Latitude } \\ \text { Type } & \text { Type } & \text { Status } & \text { Status } & \text { Longitude } \\ \text { C } & \text { ROR } & \text { XX } & \text { WO } & \end{array}$

Factor

Exist Prob

Factor

Exist Prob

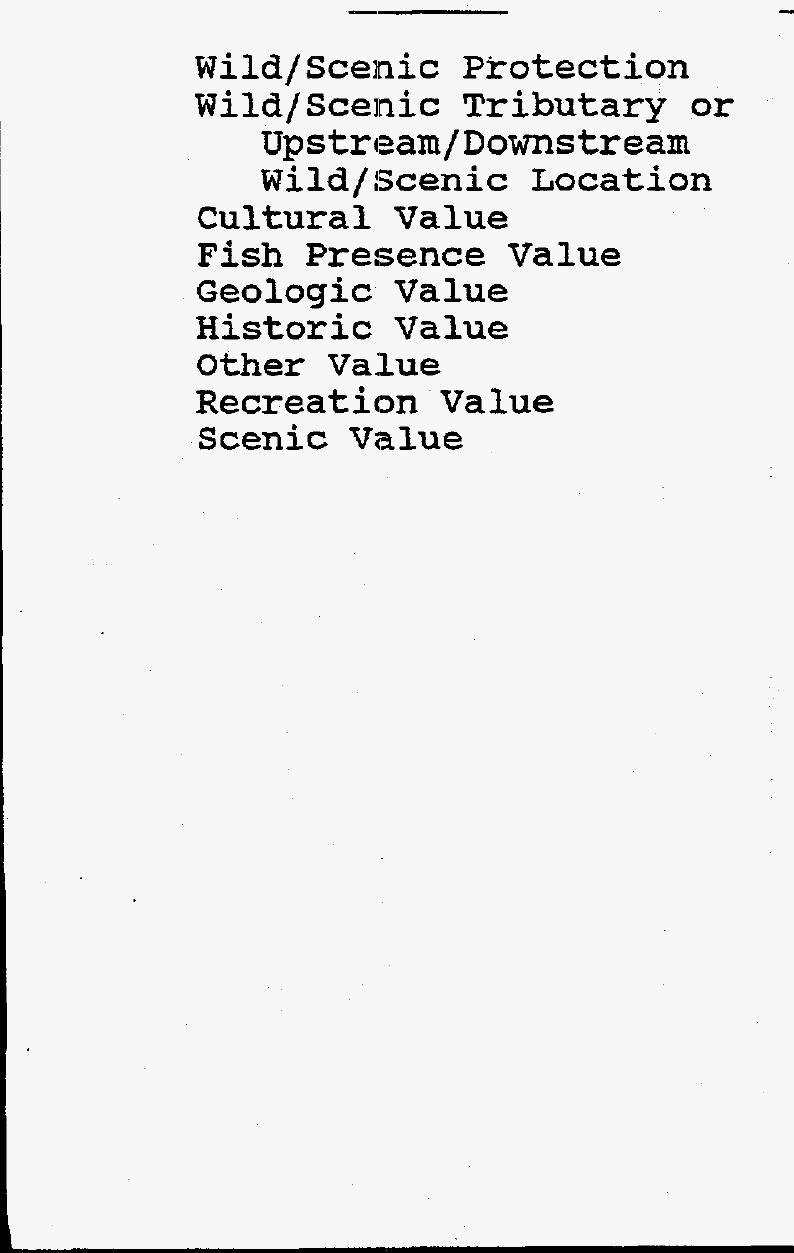

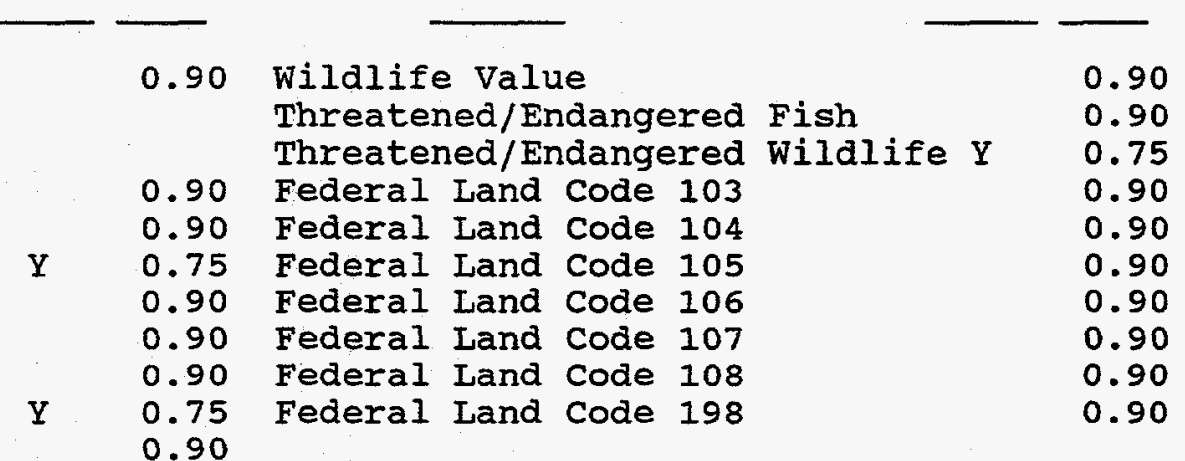


DATE: $05 / 23 / 97$

PAGE NO: 15

FERC

Number

Plant Name

Stream

state

11351 OLD COLUMBIA

DUCK $\mathbf{R}$

Name

county Name

River Basin

MAURY

TENNESSEE RIVER BASIN

$\begin{array}{cr}\text { Class } & \text { Owner Name } \\ \text { M } & \text { CITY OF COLUMBIA }\end{array}$

$\begin{array}{lcccc}\begin{array}{l}\text { Name Plate } \\ \text { Rating (KW) }\end{array} & \text { PESF } & \text { PESF*KW } & \begin{array}{l}\text { Annual Energy } \\ \text { Rating (MWh) }\end{array} & \begin{array}{c}\text { PESF Annual } \\ \text { Energy Rating (MWh) }\end{array} \\ 800.00 & 0.50 & 400.00 & 4000.00 & 2000\end{array}$

$\begin{array}{lllll}\text { Unit } & \text { Plant } & \text { Project } & \text { Dam } & \text { Latitude } \\ \text { Type } & \text { Type } & \text { Status } & \text { Status } & \text { Longitude } 8701.00\end{array}$

C ROR NO WO

Factor

Wild/scenic Protection

wild/Scenic Tributary or

Upstream/Downstream

wild/Scenic Location

Cultural value

Fish Presence Value

Geologic Value

Historic Value

other Value

Recreation value

Scenic Value
Exist Prob



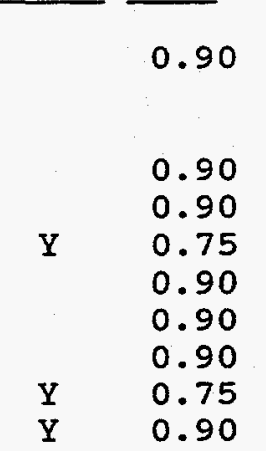

\section{Factor}

Exist Prob

Wildife Value Threatened/Endangered Fish Threatened/Endangered Wildlife $\mathrm{Y}$ Federal Land Code 103

Federal Land Code 104

Federal Land Code 105

Federal Land Code 106

Federal Land Code 107

Federal Land Code 108

Federal Land Code 198
TN 
DATE: $05 / 23 / 97$

PAGE NO: 16

FERC

Number

Plant Name

Stream

state

TNO01 COIUMBIA

DUCK $\mathbf{R}$

Name

TNOO1 COLUMBIA

County Name

River Basin

MAURY

TENNESSEE RIVER BASIN

Class Owner Name

F TENNESSEE VALLEY AUTH

Name Plate

PESF PESF*KW Rating (MWh)

PESF Annual

Rating (KW)

$0.10 \quad 2178.00$

52750.00

5275

21780.00

Plant Project

status

Dam
status

Latitude

Longitude

TN

\begin{tabular}{|c|c|c|c|c|c|}
\hline $\begin{array}{l}\text { Unit } \\
\text { Type }\end{array}$ & $\begin{array}{l}\text { Plant } \\
\text { Type }\end{array}$ & $\begin{array}{l}\text { Project } \\
\text { status }\end{array}$ & $\begin{array}{l}\text { Dam } \\
\text { status }\end{array}$ & $\begin{array}{l}\text { Latitude } \\
\text { Longitude }\end{array}$ & $\begin{array}{l}3536.00 \\
8700.00\end{array}$ \\
\hline c & ROR & & $\mathrm{U}$ & & \\
\hline
\end{tabular}

Factor

Exist Prob

Factor

Exist Prob

1d/Scenic Protection

Wild/Scenic Tributary or Upstream/ Downstream wild/Scenic Location Cultural Value

Fish Presence Value

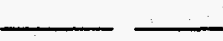

Wildlife Value

0.90

Threatened/Endangered Fish

0.90

Threatened/Endangered Wildlife $Y$

0.50

0.90 Federal Land code 103

0.90

0.90 Federal Iand code 104

0.90

$\mathrm{Y}$

0.25 Federal Land code 105

0.90

Geologic Value

0.90

Federal Land Code 106

0.90

0.90 Federal Land code 107

0.90

other Value

Recreation Value

0.90

Federal Land Code 108

0.90

$\begin{array}{ll}\mathrm{Y} & 0.25 \\ \mathrm{Y} & 0.50\end{array}$

Federal Land Code 198

0.90 
FERC

Number

Plant Name

TNO02 NORMANDY

county Name

COFFEE stream

DUCK $\mathbf{R}$

River Basin

TENNESSEE RIVER BASIN
State

Name

TN

$$
\begin{array}{cc}
\text { Class } & \text { Owner Name } \\
\text { F } & \text { TENNESSEE VALLEY AUTH }
\end{array}
$$

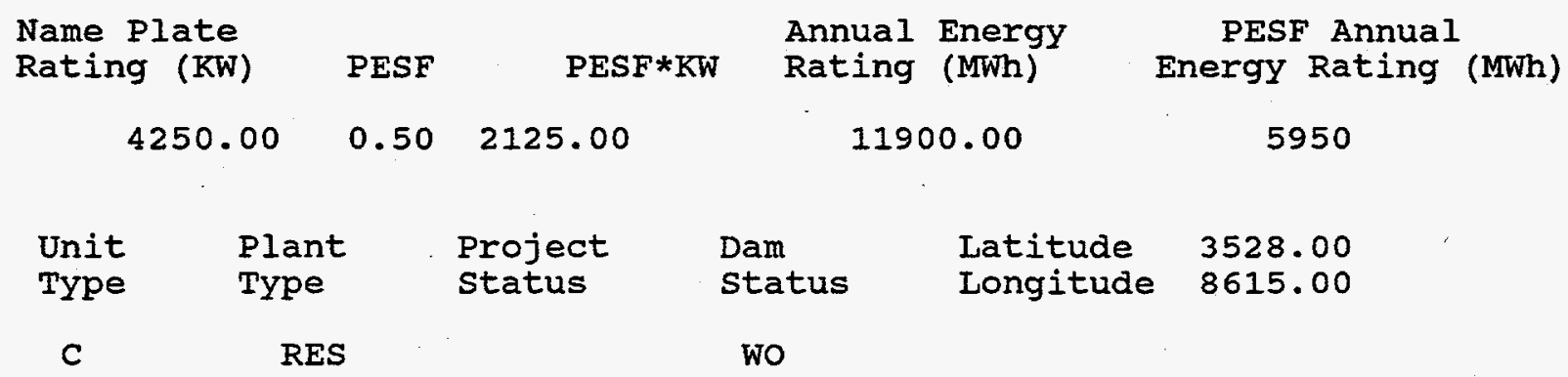

\section{Factor}

wild/Scenic Protection Wild/Scenic Tributary or Upstream/Downstream wild/Scenic Location Cultural Value

Fish Presence Value Geologic Value Historic Value other Value Recreation value Scenic Value
Exist Prob

Factor

Exist Prob

$$
0.90
$$

Wildlife Value Threatened/Endangered Fish Threatened/Endangered Wildlife Y

0.90

0.90 Federal Land code 103

0.90 Federal Land Code 104

$Y \quad 0.75$ Federal Land code 105

0.90 Federal Land code 106

0.90 Federal Land Code 107

0.90 Federal Land Code 108

$Y \quad 0.75$ Federal Land code 198

$\mathrm{Y} \quad 0.90$
0.90

0.75

0.90

0.90

0.90

0.90

0.90

0.90

0.90 
R E S O U R E D A T A B A S E L I S T I N G

DATE: $05 / 23 / 97$

PAGE NO: 18

FERC

Number

Plant Name

Stream

State

TNO03

CUMBERLAND GAP

POWELI R

Name

\section{$1 \mathrm{NoO}$}

county Name

River Basin

CLAIBORNE

TENNESSEE RIVER BASIN

$\begin{array}{cc}\text { Class } & \text { Owner Name } \\ \text { F } & \text { TENNESSEE VALLEY AUTH }\end{array}$

$\begin{array}{lll}\text { Name Plate } & & \text { Annual Energy } \\ \text { Rating (KW) PESF } & \text { PESF*KW } & \text { Rating (MWh) }\end{array}$

71400.00

$0.10 \quad 7140.00$

117100.00

Dam Latitude 3633.00

$\begin{array}{lllll}\text { Unit } & \text { Plant } & \text { Project } & \text { Dam } & \text { Latitude } \\ \text { Type } & \text { Type } & \text { Status } & \text { Status } & \text { Longitude } 8338.00\end{array}$

C ROR

Factor

Exist Prob

Factor

Exist Prob

Wild/Scenic Protection

Wild/Scenic Tributary or Upstream/Downstream wild/Scenic Location Cultural value

Fish Presence Value

Geologic Value

Historic Value

other value

Recreation Value

Scenic Value
-

$\mathrm{Y} \quad 0.10$

Wildlife Value

Threatened/Endangered Fish

Threatened/Endangered Wildiife $\mathrm{Y}$

$Y$

0.25

0.90

0.50

0.90 Federal Land Code 103

$Y \quad 0.50$ Federal Land code 104

$Y \quad 0.25$ Federal Land Code 105

$Y \quad 0.50$ Federal Land Code 106

$Y \quad 0.50$ Federal Iand code 107

0.90 Federal Land code 108

$Y \quad 0.25$ Federal Land code 198

$\mathrm{Y} \quad 0.50$
0.90

0.90

0.90

0.90

0.90

0.90

0.90 
FERC

Number

Plant Name

TNOO4

WAR RIDGE

County Name

CLAIBORNE

$$
\begin{array}{cc}
\text { Class } & \text { Owner Name } \\
\text { F } & \text { TENNESSEE VALIEY AUTH }
\end{array}
$$

River Basin
State

Name

CLINCH R

TN

TENNESSEE RIVER BASIN

\begin{tabular}{|c|c|c|c|c|}
\hline $\begin{array}{l}\text { Name Plate } \\
\text { Rating (KW) }\end{array}$ & PESF & PESF*KW & $\begin{array}{l}\text { Annual Energy } \\
\text { Rating (MWh) }\end{array}$ & $\begin{array}{l}\text { PESF Annual } \\
\text { Energy Rating (MWh) }\end{array}$ \\
\hline 113300.00 & 0.10 & 11330.00 & 209400.00 & 20940 \\
\hline
\end{tabular}

PESF Annual

\begin{tabular}{|c|c|}
\hline & $\begin{array}{l}\text { Plant } \\
\text { Type }\end{array}$ \\
\hline
\end{tabular}

C STG U

Factor

Wild/Scenic Protection

wild/Scenic Tributary or Upstream/Downstream wild/Scenic Location Cultural Value Fish Presence Value Geologic Value Historic Value other value Recreation value Scenic Value
Exist Prob

$\begin{array}{ll} & \\ & 0.90 \\ & \\ & 0.90 \\ & 0.90 \\ Y & 0.25 \\ Y & 0.50 \\ & 0.90 \\ & 0.90 \\ Y & 0.25 \\ Y & 0.50\end{array}$

Factor

Exist Prob

wildife Value Threatened/Endangered Fish Threatened/Endangered Wildlife $\mathrm{Y}$ Federal Land code 103 Federal Land Code 104 Federal Land Code 105 Federal Land Code 106 Federal Land code 107 Federal Land Code 108 Federal Land Code 198

\begin{tabular}{cl} 
& \\
\cline { 2 - 2 }$Y$ & 0.25 \\
& 0.90 \\
& 0.50 \\
& 0.90 \\
& 0.90 \\
& 0.90 \\
& 0.90 \\
& 0.90 \\
& 0.90 \\
& 0.90
\end{tabular}


DAFE: $05 / 23 / 97$

FERC

Number

TN005

LONG CREEK
Plant Name

county Name

COCKE
PAGE NO: 20

State

Name

FRENCH BROAD R

TN
River Basin

TENNESSEE RIVER BASIN

\section{Class \\ F TENNESSEE VALLEY AUTH}

$\begin{array}{lll}\text { Name Plate } & \text { Annual Energy } \\ \text { Rating (KW) } & \text { PESF } & \text { PESF*KW Rating (MWh) }\end{array}$

86100.00 0.108610 .00

217500.00

Latitude Longitude

3557.00

Unit

Plant Type STG

\section{Project} status

C

Factor

Exist Prob

Factor

Wildlife Value

wild/scenic Protection

wild/Scenic Tributary or Upstream/Downstream wild/scenic Location Cultural value Fish Presence Value Geologic Value Historic Value other value Recreation Value Scenic Value

\section{Status}

U

\begin{tabular}{ll}
\hline$Y$ & 0.10 \\
& \\
$Y$ & 0.90 \\
$Y$ & 0.50 \\
$Y$ & 0.25 \\
$Y$ & 0.50 \\
$Y$ & 0.50 \\
$Y$ & 0.50 \\
$Y$ & 0.25 \\
$Y$ & 0.50
\end{tabular}
Threatened/Endangered Fish Threatened/Endangered Wildiife Federal Land Code 103 Federal Land Code 104 Federal Land code 105 Federal Land Code 106 Federal Land Code 107 Federal Land Code 108 Federal Land Code 198
PESF Annual Energy Rating (MWh)

$$
21750
$$
Exist Prob

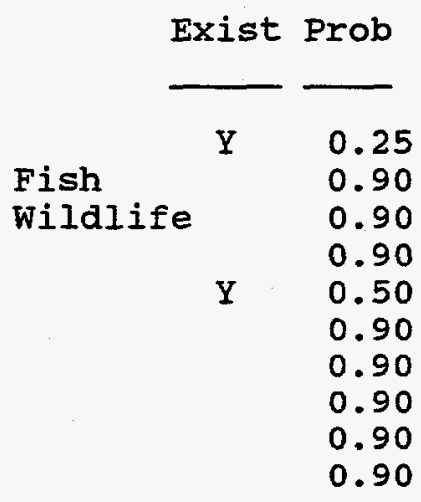


R E S O U C E D A T A B A S E

DATE: $05 / 23 / 97$

FERC

Number

Plant Name

TN006

PINE CAMP

county Name

CARTER

class

$\mathbf{F}$

TENNESSEE VALLEY AUTH

Name Plate

Rating (KW)

11820.00

PESF

PESF*KW

Annual Energy

Rating (MWh)

32400.00

$0.10 \quad 1182.00$

ELK R

Stream

Name state

TN

PESF Annual Energy Rating (MWh)

3240

PAGE NO: 21

$\begin{array}{cclll}\text { Unit } & \text { Plant } & \text { Project } & \text { Dam } & \text { Latitude } \\ \text { Type } & \text { Type } & \text { Status } & \text { Status } & \text { Longitude } 8158.00 \\ \text { C } & \text { DIV } & & \mathrm{U} & \end{array}$

Factor

wild/scenic Protection

wild/Scenic Tributary or Upstream/Downstream Wild/Scenic Location Cultural Value

Fish Presence Value

Geologic Value

Historic Value

other Value

Recreation Value

Scenic Value
Exist Prob

Factor

Exist Prob

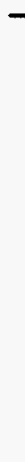

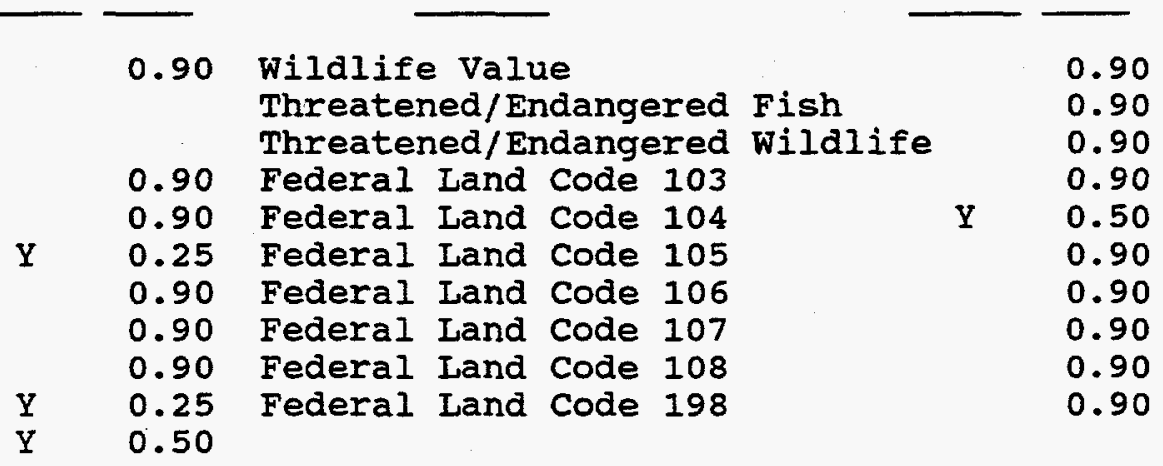


FERC

Number

TNO07
Plant Name

CANE CREEK

County Name

LEWIS
Stream

S FK CANE CR, TENNESSEE $R$

River Basin

TENNESSEE RIVER BASIN

\section{class owner Name}

$R$ WHITEHEAD, DEBRA

Name Plate Rating (KW)

Annual Energy PESF Rating (MWh)

PESF Annual

10.00

$$
0.90 \quad 9.00
$$

44.00

39.60

$\begin{array}{cclll}\text { Unit } & \text { Plant } & \text { Project } & \text { Dam } & \text { Latitude } \\ \text { Type } & \text { Type } & \text { Status } & \text { Status } & \text { Longitude } 8737.00 \\ \text { C } & \text { ROR } & & \text { U } & \end{array}$

Factor

Wild/scenic Protection

Wild/Scenic Tributary or Upstream/Downstream wild/Scenic Location Cultural Value Fish Presence Value Geologic Value Historic Value other Value Recreation Value Scenic Value
Exist Prob

0.90

Wildife Value Threatened/Endangered Fish Threatened/Endangered Wildilfe

0.90 Federal Iand code 103

0.90 Federal Iand code 104

0.90 Federal Land code 105

0.90 Federal Land code 106

0.90 Federal Land code 107

0.90 Federal Land code 108

0.90 Federal Iand code 198

0.90 state

Name

TN Energy Rating (MWh) 